Supporting Information

\title{
Further Investigations of Racemic and Chiral Molecular Sieves of STW Topology.
}

\author{
Jong Hun Kang ${ }^{\mathrm{a}, \mathrm{b}}$, Lynne B. McCusker ${ }^{\mathrm{c}}$, Michael W. Deem ${ }^{\mathrm{d}}$, Christian Baerlocher ${ }^{\mathrm{e}}$ \\ and Mark E. Davis ${ }^{\mathrm{a}, *}$
}

${ }^{a}$ Chemical Engineering, California Institute of Technology, Pasadena, California, United States 91125

${ }^{b}$ School of Chemical and Biological Engineering, and Institute of Chemical Processes, Seoul National University, Seoul, Republic of Korea 08826

'Department of Materials, ETH Zurich, Vladimir-Prelog-Weg 1-5/10, CH-8093 Zurich, Switzerland

${ }^{\mathrm{d}}$ Department of Bioengineering and Physics \& Astronomy, Rice University, Houston, Texas, United States 77005

eDepartment of Chemistry and Applied Biosciences, ETH Zurich, Vladimir-Prelog-Weg 1-5/10, CH-8093

Zurich, Switzerland

* e-mail:mdavis@cheme.caltech.edu 


\section{Table of Contents}

Synthesis and Characterization of STW Using 12345PMI Monoquat OSDA with Amino Acid Additives 3

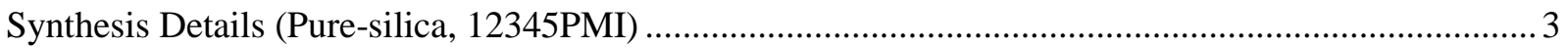

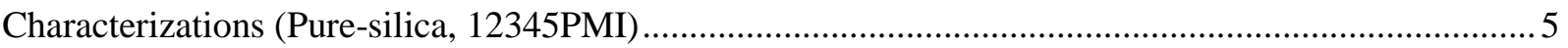

Synthesis Details (Pure-silica, 12345PMI, Influences of Fluoride Concentration in Gels)....................7

Synthesis and Characterization of STW Using 2E134TMI Monoquat OSDA with Amino Acid Additives 9

Synthesis Details (Pure-silica, 2E134TMI) ....................................................................................... 9

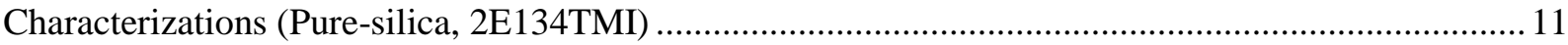

Synthesis and Characterization (Pure-silica, 2E134TMI-12345PMI 1:1 Mixed) ................................. 12

Crystallographic Information for Pure-silica STW Containing 12345PMI and 2E134TMI Monoquaternary

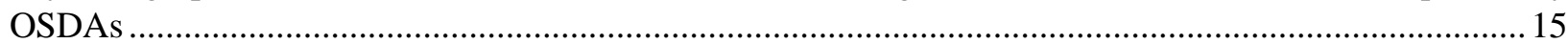

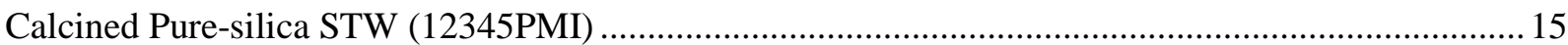

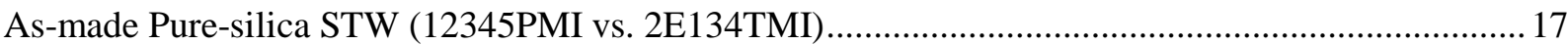

Synthesis and Characterization of Enantioenriched Germanosilicate R-STW Using Chiral Diquat OSDA

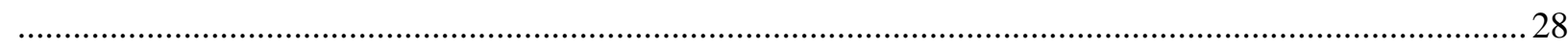

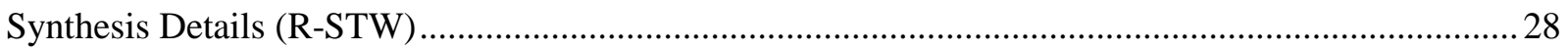

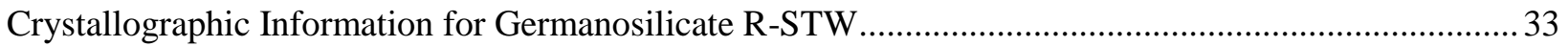

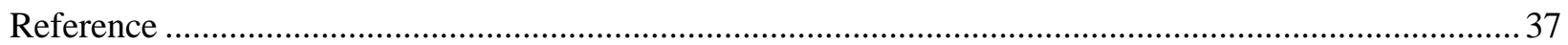




\section{Synthesis and Characterization of STW Using 12345PMI Monoquat OSDA with Amino Acid Additives}

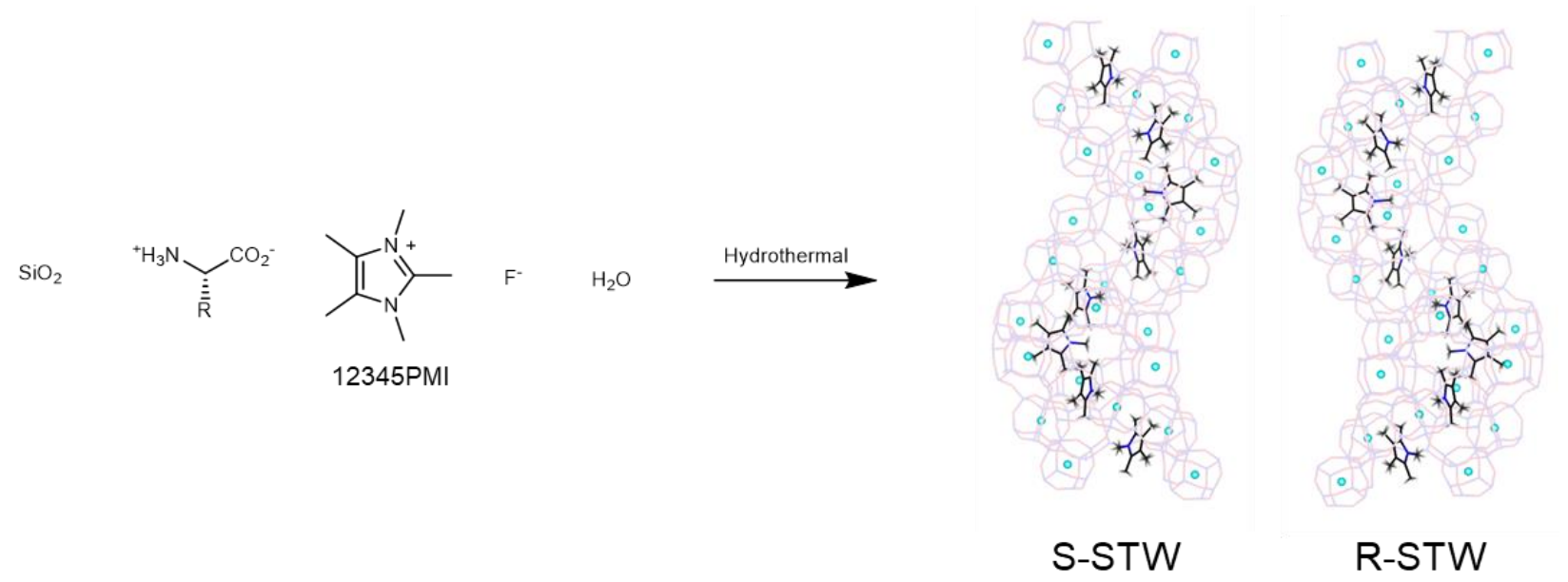

Figure S1. Schematic illustration of the synthesis of pure-silica racemic STW from 12345PMI OSDA with amino-acid additive.

\section{Synthesis Details (Pure-silica, 12345PMI)}

All chemicals were used without further purification steps. Pure-silica STW molecular sieves using 12345PMI (pentamethylimidazolium, denoted as 'R1' in gel compositions and the sample nomenclature) OSDA were synthesized by the hydrothermal synthesis using hydrofluoric acid (HF) as the mineralizer. 12345PMI hydroxide solution was prepared according to our previous report. (1) The gel composition formula was $1.0 \mathrm{SiO}_{2}: 0.5 \mathrm{R}_{1} \mathrm{OH}: x$ Amino Acid : $0.5+x \mathrm{HF}: y \mathrm{H}_{2} \mathrm{O}$, and the values of $\mathrm{x}$ and $\mathrm{y}$ and other synthesis parameters are shown in Table S1. For example, in the case of the synthesis of R1-P2 which was used for the crystallographic study, $0.108 \mathrm{~g}(0.941 \mathrm{mmol})$ or L-proline (Sigma-Aldrich) was fully dissolved in $5.96 \mathrm{~g}$ of $12345 \mathrm{PMI}$ hydroxide solution $\left(0.395 \mathrm{mmol} / \mathrm{g}\right.$ in $\left.\mathrm{H}_{2} \mathrm{O}\right)$ in a $23-\mathrm{mL}$ PTFE liner of Parr autoclave. $1.000 \mathrm{~g}(4.70 \mathrm{mmol})$ of tetraethyl orthosilicate (TEOS, Alfa Aesar, 99.9\%) was added to the mixture and hydrolyzed by stirring overnight. The access amount of water was evaporated under an airflow. After the gel became viscous, $0.137 \mathrm{~g}$ (3.29 mmol HF) of HF solution (Sigma-Aldrich, $48 \%$ in $\mathrm{H}_{2} \mathrm{O}$ ) was added and mechanically mixed using a PTFE rod. The resultant white powdery gel was further dried overnight in a fume hood. Finally, a desired amount of distilled water was added to make $y=7$, and the gel was tightly sealed in a Parr steel autoclave and placed in a rotating oven at $160{ }^{\circ} \mathrm{C}$. The progress of crystallization was monitored by taking aliquots weekly. The finished batch was washed with distilled water and acetone, and dried at $100{ }^{\circ} \mathrm{C}$ overnight. 
Table S1. Summary of 12345PMI-based pure-silica STW synthesis with amino-acid additives (gel composition: $1.0 \mathrm{SiO}_{2}: 0.5 \mathrm{R}_{1} \mathrm{OH}: x$ Amino Acid : $0.5+x \mathrm{HF}: y \mathrm{H}_{2} \mathrm{O}$ ).

\begin{tabular}{|c|c|c|c|c|c|}
\hline \multirow{2}{*}{ R1-\# } & \multicolumn{2}{|c|}{ Amino Acid } & \multirow{2}{*}{$\begin{array}{c}\text { Condition } \\
\mathbf{T}\left({ }^{\circ} \mathbf{C}\right)\end{array}$} & \multirow{2}{*}{ Time (days) } & \multirow{2}{*}{ Result } \\
\hline & Type* & $x$ & & & \\
\hline 00 & - & - & 160 & 14 & STW \\
\hline V1 & $\bar{V}$ & 0.1 & 160 & 7 & STW \\
\hline $\mathrm{V} 2$ & V & 0.2 & 160 & & STW \\
\hline $\mathrm{V} 2 * \dagger$ & $\mathrm{V}$ & $0.2^{\dagger}$ & 160 & & STW \\
\hline V3 & $\mathrm{V}$ & 0.3 & 160 & 14 & STW+? \\
\hline V5 & $\mathrm{V}$ & 0.5 & 160 & 14 & $?$ \\
\hline M1 & $\bar{M}$ & 0.1 & 160 & 14 & $\mathrm{STW}+7.9^{\circ}$ \\
\hline M2 & M & 0.2 & 160 & 14 & $?$ \\
\hline$\overline{S 1}$ & $\bar{S}$ & 0.1 & 160 & 14 & $\mathrm{STW}+7.9^{\circ}$ \\
\hline S2 & $S$ & 0.2 & 160 & 7 & STW \\
\hline S3 & $S$ & 0.3 & 160 & 14 & $?$ \\
\hline L1 & $\bar{L}$ & 0.1 & 160 & 7 & $\overline{\text { STW }}$ \\
\hline L1-175 & $\mathrm{L}$ & 0.1 & 175 & & \\
\hline L2 & $\mathrm{L}$ & 0.2 & 160 & 7 & STW \\
\hline L3 & $\mathrm{L}$ & 0.3 & 160 & 14 & STW+? \\
\hline L4 & $\mathrm{L}$ & 0.4 & 160 & 7 & $?$ \\
\hline A1-175 & $\mathrm{A}$ & 0.1 & 175 & 14 & $\overline{\text { STW }}$ \\
\hline A1 & A & 0.1 & 160 & 7 & STW \\
\hline $\mathrm{A} 2$ & A & 0.2 & 160 & 14 & STW \\
\hline P1-175 & $\bar{P}$ & 0.1 & 175 & 7 & $\mathrm{STW}+7.9^{\circ}$ \\
\hline P1 & $\mathrm{P}$ & 0.1 & 160 & 7 & STW \\
\hline P2 & $\mathrm{P}$ & 0.2 & 160 & 7 & STW \\
\hline P3 & $\mathrm{P}$ & 0.3 & 160 & 14 & $?$ \\
\hline I1 & I & 0.1 & 160 & 7 & $\overline{\text { STW }}$ \\
\hline $\mathrm{I} 1-175$ & I & 0.1 & 175 & & \\
\hline I2 & I & 0.2 & 160 & 7 & STW \\
\hline $\mathrm{I} 3$ & I & 0.3 & 160 & 7 & \\
\hline I4 & I & 0.4 & 160 & 7 & \\
\hline$\overline{F 1}$ & $\bar{F}$ & 0.1 & 160 & 7 & STW \\
\hline $\mathrm{F} 2$ & $\mathrm{~F}$ & 0.2 & 160 & 7 & STW \\
\hline
\end{tabular}

\footnotetext{
${ }^{*}$ One-letter abbreviations were used $(\mathrm{V}=$ valine, $\mathrm{M}=$ methionine, $\mathrm{S}=$ serine, $\mathrm{L}=$ leucine, $\mathrm{A}=$ alanine, $\mathrm{P}=$ proline, $\mathrm{I}$ $=$ isoleucine, $\mathrm{F}=$ phenylalanine). ${ }^{\top}$ The gel composition for this batch is $1.0 \mathrm{SiO}_{2}: 0.5 \mathrm{R}_{1} \mathrm{OH}: \mathrm{x}$ Amino Acid : $0.5 \mathrm{HF}$ : y $\mathrm{H}_{2} \mathrm{O}$ (i.e., extra $\mathrm{HF}$ for the complete protonation of amino acid was not added to this batch.). $\$ 7.9^{\circ}$ denotes an unknown impurity that show a small peak at $2 \theta=7.9^{\circ}$.
} 


\section{Characterizations (Pure-silica, 12345PMI)}

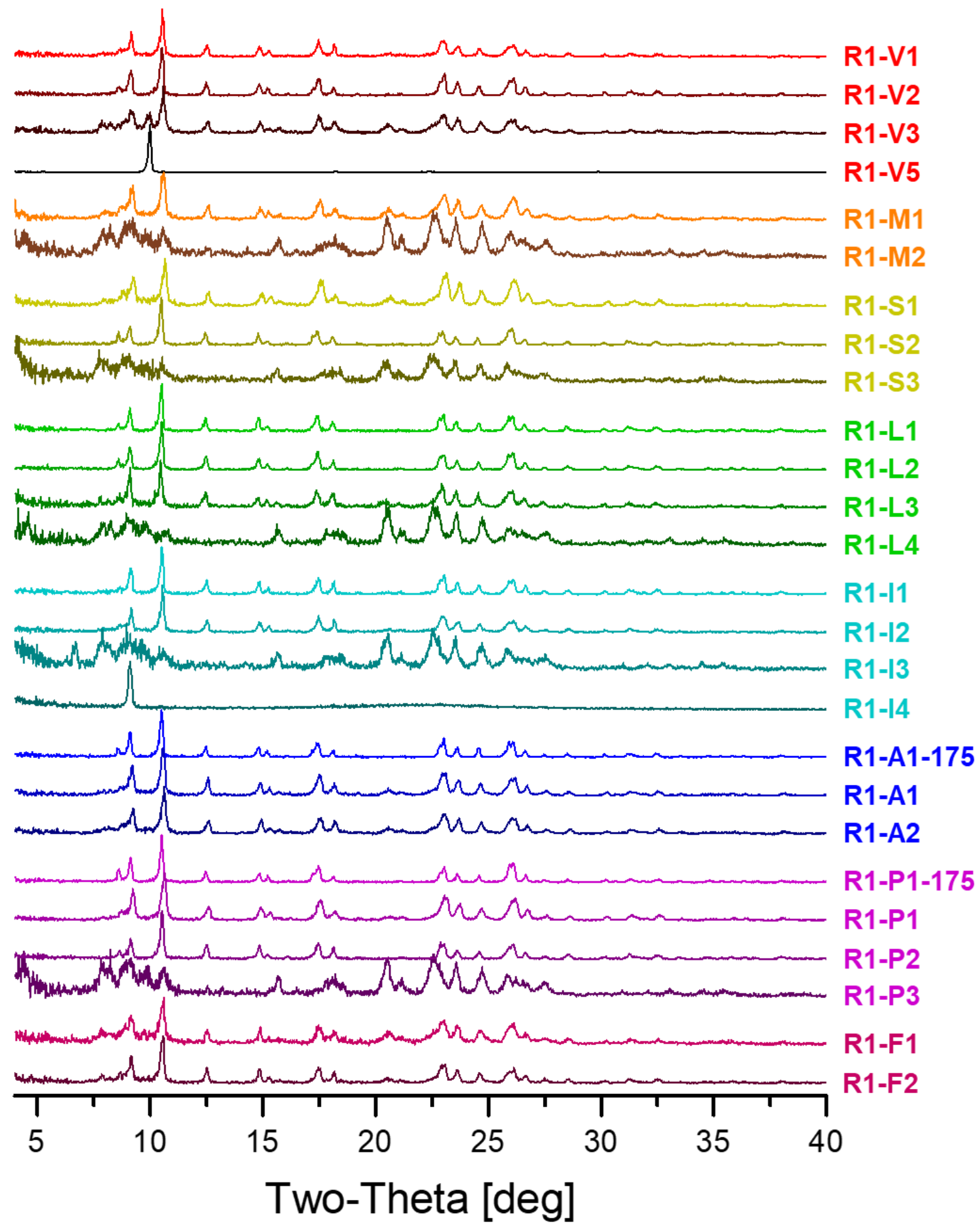

Figure S2. PXRD profiles of crystallization products obtained from the 12345PMI-based pure-silica STW syntheses with various amino-acid additives of different concentrations 


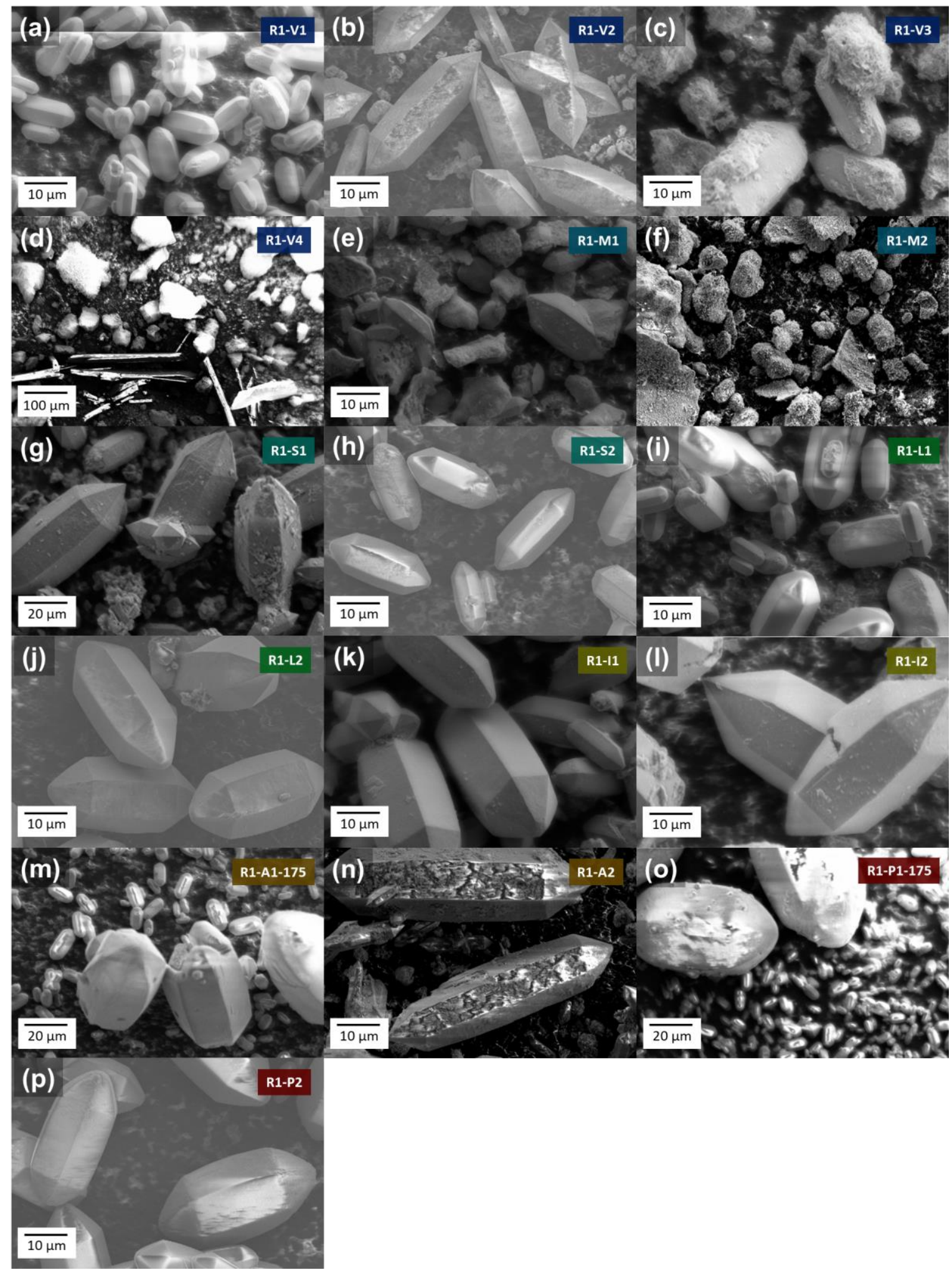

Figure S3. SEM images of crystals obtained from the 12345PMI-based pure-silica STW syntheses with various amino-acid additives of different concentrations: (a) R1-V1, (b) R1-V2, (c) R1-V3, (d) R1-V5, (e) R1-M1, (f) R1-M2, (g) R1-S1, (h) R1-S2, (i) R1-L1, (j) R1-L2, (k) R1-I1, (l) R1-I2, (m) R1-A1-175, (n) R1-A2, (o) R1-P1-175, and (p) R1-P2. 


\section{Synthesis Details (Pure-silica, 12345PMI, Influences of Fluoride Concentration in Gels)}

The concentration of fluoride in synthesis gels was controlled separately from the amino-acid additive concentration, by adding ammonium fluoride. The procedures were analogous to the cases of pure-silica syntheses shown above. L-leucine (L) was selected as the additive for the aluminosilicate syntheses. The gel composition formula was $1.0 \mathrm{SiO}_{2}: 0.5 \mathrm{R}_{1} \mathrm{OH}: x \mathrm{~L}: y \mathrm{HF}: z \mathrm{NH}_{4} \mathrm{~F}: 7.0 \mathrm{H}_{2} \mathrm{O}$, and the values of $x, y, z$, and other synthesis parameters are shown in Table $\mathrm{S} 2$.

Table S2. Summary of 12345PMI-based pure-silica STW synthesis with varied levels of L-leucine and fluoride concentrations. (gel composition: $1.0 \mathrm{SiO}_{2}: 0.5 \mathrm{R}_{1} \mathrm{OH}: x \mathrm{~L}: y \mathrm{HF}: z \mathrm{NH}_{4} \mathrm{~F}: 7.0 \mathrm{H}_{2} \mathrm{O}$ ).

\begin{tabular}{|c|c|c|c|c|c|c|c|c|c|}
\hline \multirow{2}{*}{ R1-\# } & \multicolumn{2}{|c|}{ Amino Acid } & \multirow[b]{2}{*}{$y$} & \multirow{2}{*}{$z$} & \multirow{2}{*}{$\begin{array}{c}\text { Total F } \\
(y+z)\end{array}$} & \multicolumn{2}{|c|}{ Condition } & \multirow{2}{*}{ Time (days) } & \multirow{2}{*}{ Result } \\
\hline & Type* & $\bar{x}$ & & & & $\mathbf{T}\left({ }^{\circ} \mathbf{C}\right)$ & Oven & & \\
\hline 00 & - & 0 & 0.5 & 0 & 0.5 & 160 & Rotating & 14 & STW \\
\hline $\mathrm{L} 2 *$ & $\mathrm{~L}$ & 0.2 & 0.5 & 0 & 0.5 & 160 & Rotating & 14 & STW \\
\hline AmF2 & - & 0 & 0.5 & 0.2 & 0.7 & 160 & Rotating & 14 & STW \\
\hline L2 & $\mathrm{L}$ & 0.2 & 0.7 & 0 & 0.7 & 160 & Rotating & 14 & STW \\
\hline
\end{tabular}
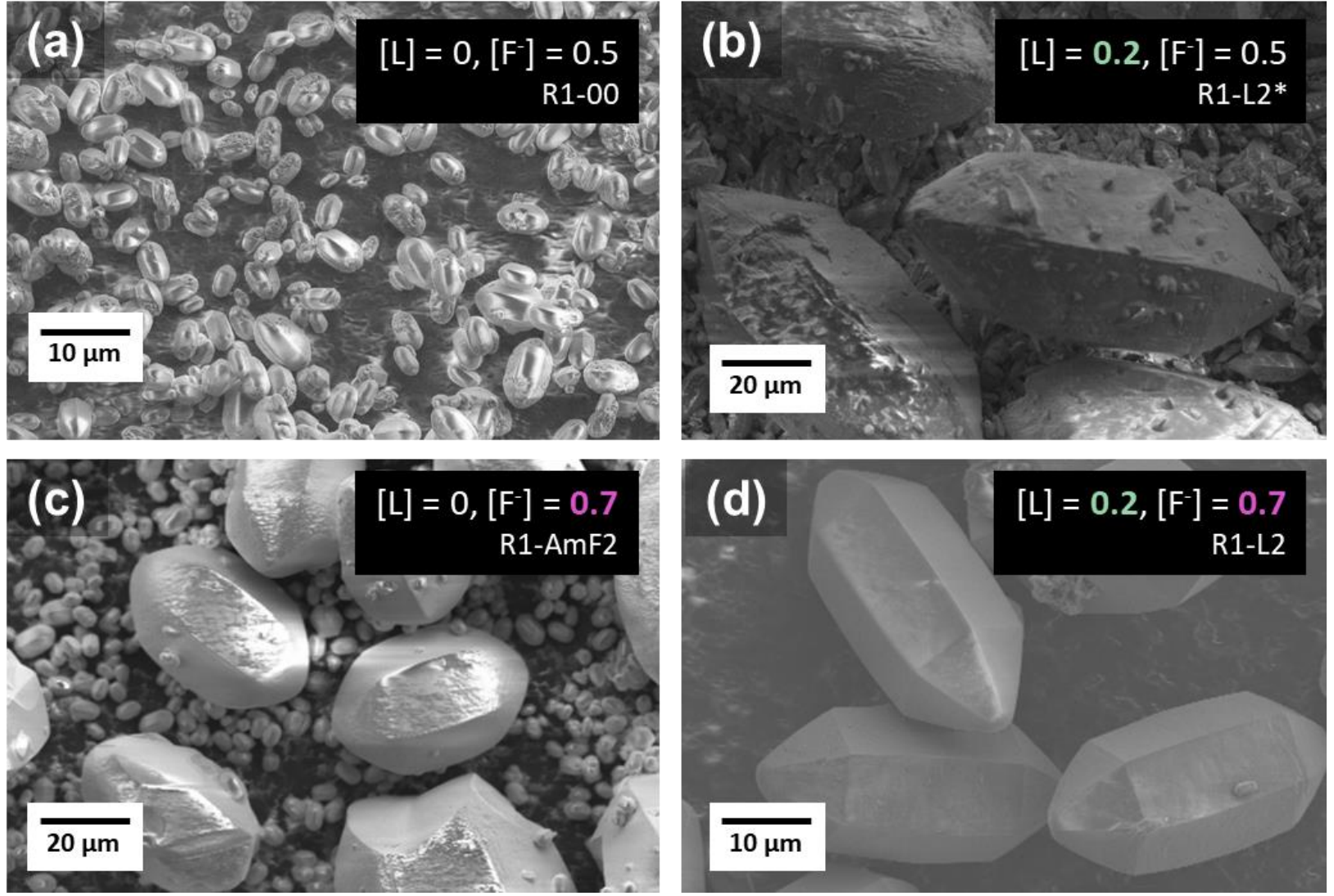

Figure S4. SEM images of crystals obtained from the 12345PMI-based pure-silica STW syntheses with individually controlled concentrations of amino-acid additives and fluoride. 


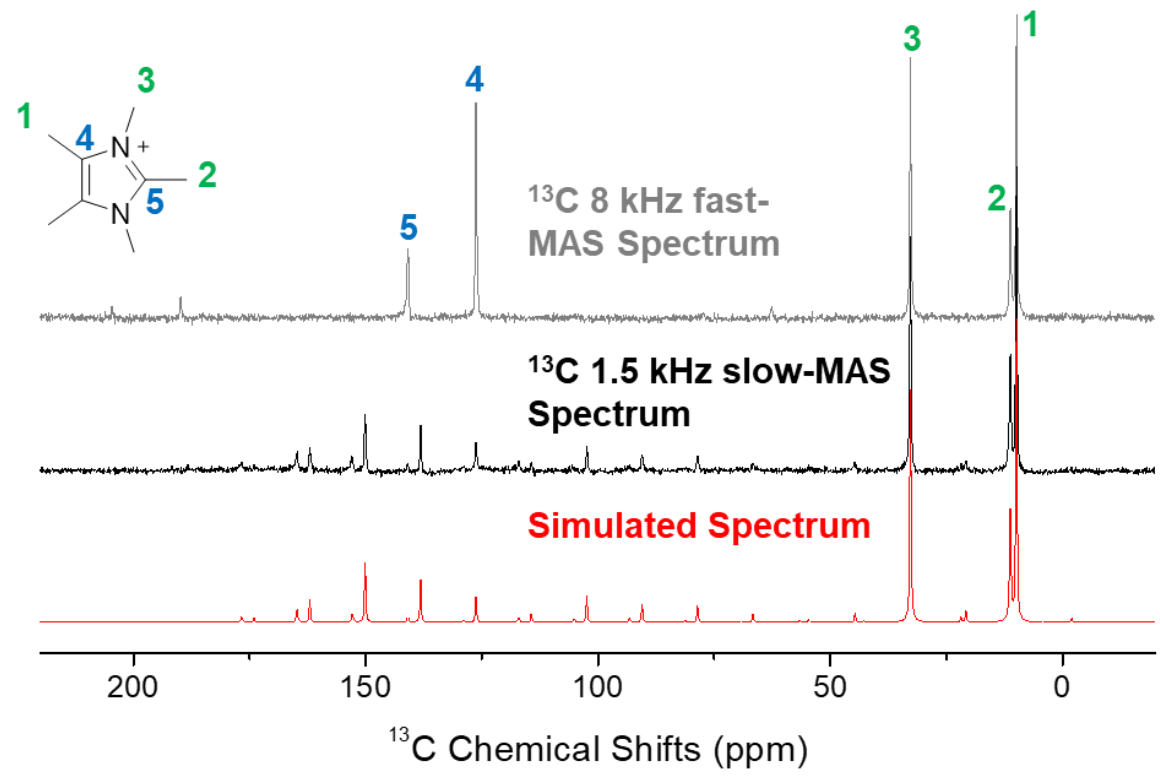

Figure S5. ${ }^{13} \mathrm{C} 8 \mathrm{kHz}$ (grey) and $1.5 \mathrm{kHz}$ (black) MAS spectra of as-made pure-silica STW that was crystallized under the presence of L-isoleucine additive. Simulated spectrum (red) was obtained using DMFIT software.

Table S3. Chemical shift anisotropy (CSA) parameters calculated from the ${ }^{13} \mathrm{C}$ slow-MAS $(1.5 \mathrm{kHz})$ spectrum (red spectrum of Figure S5) of as-made pure-silica STW crystalized under the presence of Lisoleucine.

\begin{tabular}{ccccccccc} 
Peak \# & Amplitude & $\begin{array}{c}\text { Carbon } \\
\text { Type }\end{array}$ & $\begin{array}{c}\text { Isotropic } \\
\text { Shift }\left(\delta_{\text {iso }},\right. \\
\mathrm{ppm})\end{array}$ & $\begin{array}{c}\text { Reduced } \\
\text { Anisotropy } \\
(\mathrm{dCS}, \mathrm{ppm})\end{array}$ & & \multicolumn{3}{c}{$\begin{array}{c}\text { Anisotropic Tensor Principal } \\
\text { Components }(\mathrm{ppm})\end{array}$} \\
\hline 1 & 27477 & Methyl & 9.92 & -5.89 & 0.037 & 4.03 & 12.76 & 12.97 \\
2 & 10040 & & 11.24 & -0.81 & 0.039 & 10.43 & 11.63 & 11.66 \\
3 & 20981 & & 32.74 & 9.73 & 0.015 & 42.47 & 27.95 & 27.80 \\
\hline 4 & 2323 & Aromatic & 126.28 & -67.15 & 0.110 & 59.13 & 156.16 & 163.55 \\
5 & 441 & Heterocycle & 140.93 & -69.88 & 0.013 & 71.05 & 175.42 & 176.32
\end{tabular}




\section{Synthesis and Characterization of STW Using 2E134TMI Monoquat OSDA with Amino Acid Additives}

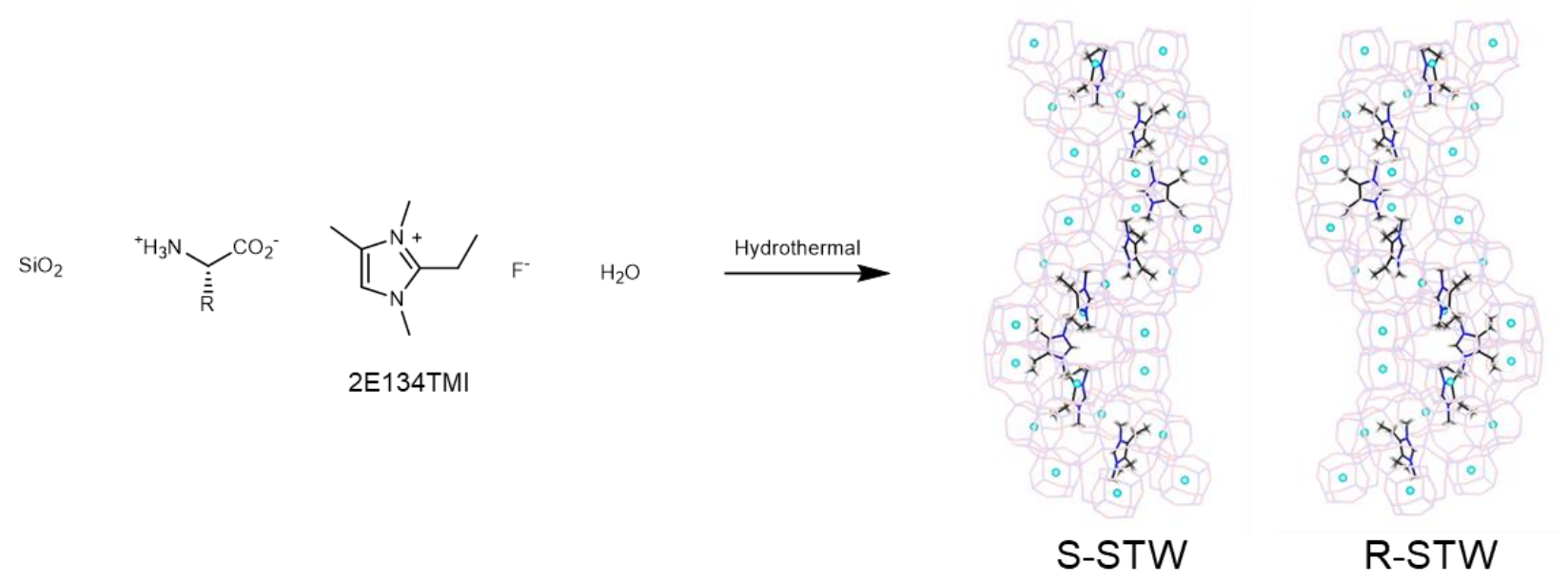

Figure S6. Schematic illustration of the synthesis of pure-silica racemic STW from 2E134TMI OSDA with amino-acid additive.

\section{Synthesis Details (Pure-silica, 2E134TMI)}

All chemicals were used without further purification steps. Pure-silica STW molecular sieves using 2E134TMI (2-ethyl-1,3,4-trimethylimidazolium, denoted as 'R2' in gel compositions and the sample nomenclature) OSDA were synthesized by the hydrothermal synthesis using hydrofluoric acid (HF) as the mineralizer. 2E134TMI hydroxide solution was prepared according to Camblor's HPM-1 pure-silica STW report. (2) The gel composition formula was $1.0 \mathrm{SiO}_{2}: 0.5 \mathrm{R}_{2} \mathrm{OH}: x$ Amino Acid : 0.5+x $\mathrm{HF}: y \mathrm{H}_{2} \mathrm{O}$, and the values of $x$ and $y$ and other synthesis parameters are shown in Table $S 4$. For example, in the case of the synthesis of STW-R2-I1 which was used for the crystallographic study, $0.082 \mathrm{~g}(0.627 \mathrm{mmol})$ or Lisoleucine (Sigma-Aldrich) was fully dissolved in $2.36 \mathrm{~g}$ of 2E134TMI hydroxide solution $(1.329 \mathrm{mmol} / \mathrm{g}$ in $\left.\mathrm{H}_{2} \mathrm{O}\right)$ in a 23-mL PTFE liner of Parr autoclave. $1.333 \mathrm{~g}(6.27 \mathrm{mmol})$ of tetraethyl orthosilicate (TEOS, Alfa Aesar, 99.9\%) was added to the mixture and hydrolyzed by stirring overnight. The access amount of water was evaporated under an airflow. After the gel became viscous, $0.157 \mathrm{~g}(3.76 \mathrm{mmol} \mathrm{HF})$ of $\mathrm{HF}$ solution (Sigma-Aldrich, $48 \%$ in $\mathrm{H}_{2} \mathrm{O}$ ) was added and mechanically mixed using a PTFE rod. The resultant white powdery gel was further dried overnight in a fume hood. Finally, a desired amount of distilled water was added to make $y=4$, and the gel was tightly sealed in a Parr steel autoclave and placed in a rotating oven at $160{ }^{\circ} \mathrm{C}$. The progress of crystallization was monitored by taking aliquots weekly. The finished batch was washed with distilled water and acetone, and dried at $100{ }^{\circ} \mathrm{C}$ overnight. 
Table S4. Summary of 2E134TMI-based pure-silica STW synthesis with amino-acid additives (gel composition: $1.0 \mathrm{SiO}_{2}: 0.5 \mathrm{R}_{2} \mathrm{OH}: x$ Amino Acid : $0.5+x \mathrm{HF}: y \mathrm{H}_{2} \mathrm{O}$ ).

\begin{tabular}{|c|c|c|c|c|c|c|c|}
\hline \multirow{2}{*}{$\#$} & \multicolumn{2}{|c|}{ Amino Acid } & \multirow{2}{*}{$y$} & \multicolumn{2}{|l|}{ Condition } & \multirow{2}{*}{ Time (days) } & \multirow{2}{*}{ Result } \\
\hline & Type* & $x$ & & $\mathbf{T}\left({ }^{\circ} \mathbf{C}\right)$ & Seed & & \\
\hline R2-00-seed & - & - & 4.0 & 175 & $\mathrm{O}$ & 7 & STW \\
\hline $\mathrm{R} 2-00$ & - & - & 4.0 & 175 & $\mathrm{X}$ & 7 & STW \\
\hline R2-00-W5 & - & - & 5.0 & 175 & $\mathrm{X}$ & 7 & HPM-2 \\
\hline R2-00-W7 & - & - & 7.0 & 175 & $\mathrm{X}$ & 7 & HPM-2 \\
\hline R2-L1-175 & $\mathrm{L}$ & 0.1 & 4.0 & 175 & $\bar{X}$ & 7 & STW \\
\hline R2-L2-175 & $\mathrm{L}$ & 0.2 & 4.0 & 175 & $\mathrm{X}$ & 7 & STW+HPM-2 \\
\hline R2-L2-175-W7 & $\mathrm{L}$ & 0.2 & 7.0 & 175 & $\mathrm{X}$ & 7 & HPM-2 \\
\hline R2-L1 & $\mathrm{L}$ & 0.1 & 4.0 & 160 & $\mathrm{X}$ & 16 & STW \\
\hline R2-L2 & $\mathrm{L}$ & 0.2 & 4.0 & 160 & $\mathrm{X}$ & 16 & STW \\
\hline R2-I1 & $\bar{I}$ & 0.1 & 4.0 & 160 & $\bar{X}$ & 16 & STW \\
\hline R2-I2-175 & $\mathrm{I}$ & 0.1 & 4.0 & 175 & $\mathrm{X}$ & 16 & HPM-2 \\
\hline R2-V1 & $\overline{\mathrm{V}}$ & 0.1 & 4.0 & 160 & $\bar{X}$ & 16 & STW \\
\hline R2-V1-175 & V & 0.1 & 4.0 & 175 & $\mathrm{X}$ & 7 & STW \\
\hline R2-P1-175 & $\bar{P}$ & 0.1 & 4.0 & 175 & $\bar{X}$ & 7 & HPM-2 \\
\hline R2-P2-175 & $\mathrm{P}$ & 0.2 & 4.0 & 175 & $\mathrm{X}$ & 7 & HPM-2 \\
\hline R2-P2-175-W7 & $\mathrm{P}$ & 0.2 & 7.0 & 175 & $X$ & 7 & HPM-2 \\
\hline
\end{tabular}

${ }^{*}$ One-letter abbreviations were used $(\mathrm{V}=$ valine, $\mathrm{M}=$ methionine, $\mathrm{S}=$ serine, $\mathrm{L}=$ leucine, $\mathrm{A}=$ alanine, $\mathrm{P}=$ proline, $\mathrm{I}$ $=$ isoleucine, $\mathrm{F}=$ phenylalanine). 


\section{Characterizations (Pure-silica, 2E134TMI)}

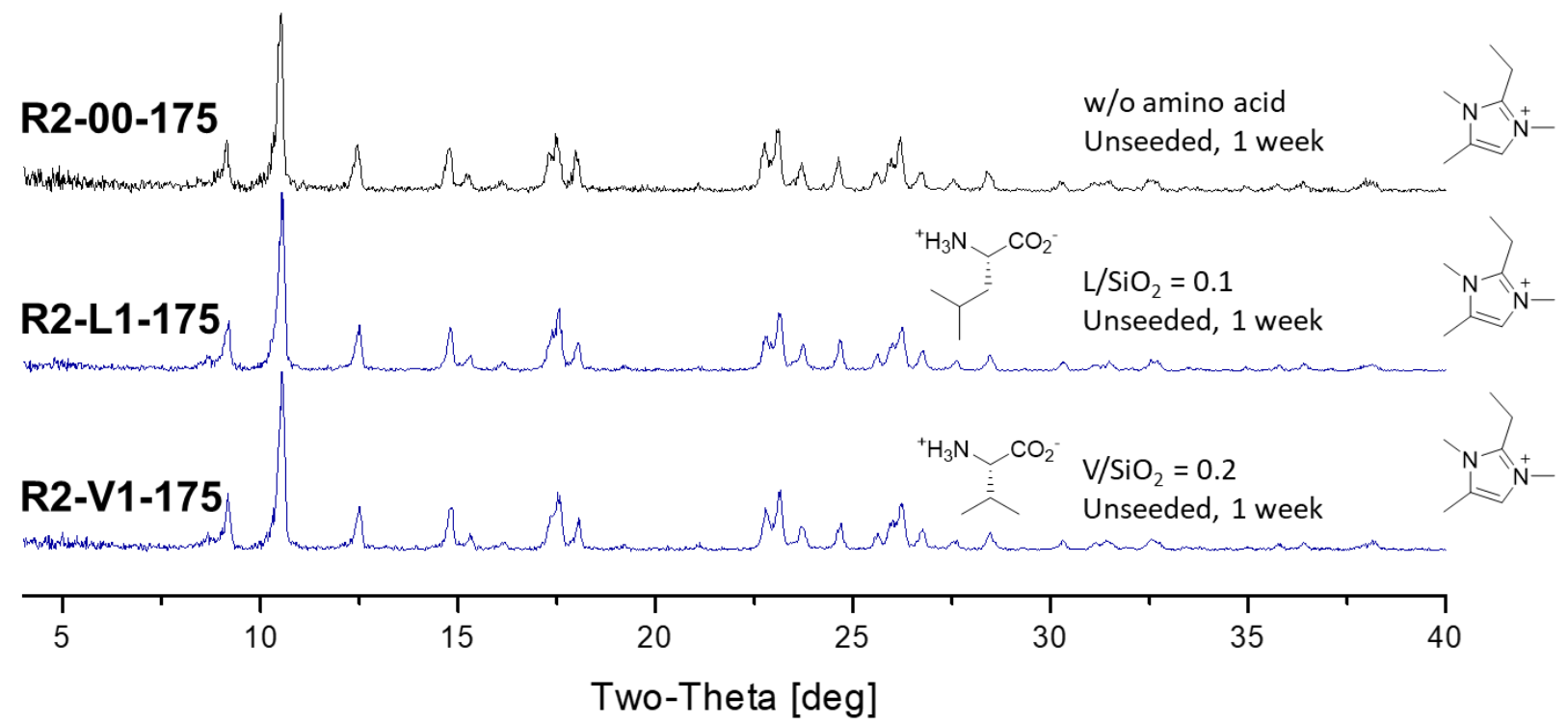

Figure S7. Selected PXRD profiles of pure-silica STW samples crystallized from the 2E134TMI OSDA system with amino acid additives.

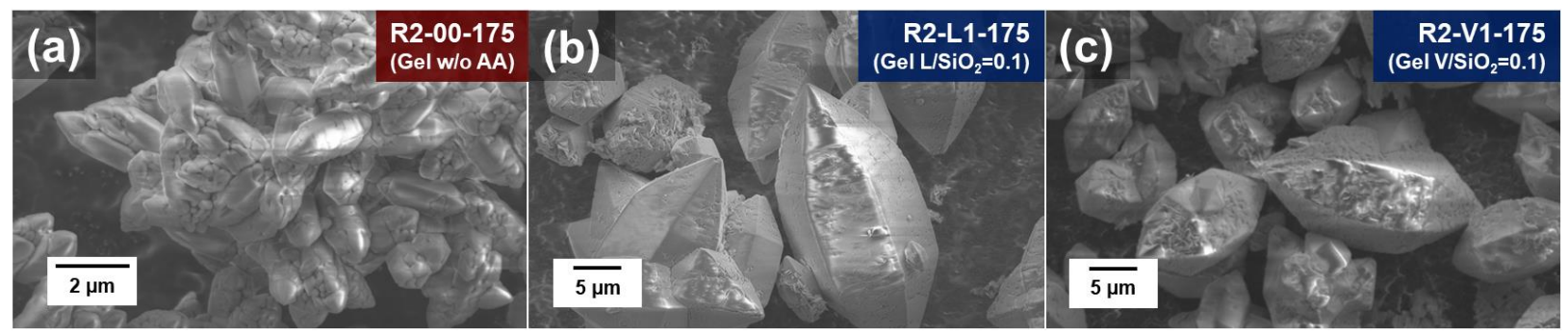

Figure S8. Scanning electron micrographs of selected pure-silica STW samples from the 2E134TMI OSDA system with amino acid additives: (a) R2-00-175, (b) R2-L1-175, and (c) R2-V1-175 


\section{Synthesis and Characterization (Pure-silica, 2E134TMI-12345PMI 1:1 Mixed)}

The procedures were analogous to the cases of pure-silica syntheses shown above. L-leucine (L) was selected as the additive for the aluminosilicate syntheses. The gel composition formula was $1.0 \mathrm{SiO}_{2}$ : $0.25 \mathrm{R}_{1} \mathrm{OH}: 0.25 \mathrm{R}_{2} \mathrm{OH}: 0.2 \mathrm{~L}$-Leucine : $0.7 \mathrm{HF}: y \mathrm{H}_{2} \mathrm{O}$, and the values of $y$ and other synthesis parameters are shown in Table S5.

Table S5. Summary of (12345PMI:2E134TMI = 1:1) mixture-based pure-silica STW synthesis with amino-acid additives (gel composition: $1.0 \mathrm{SiO}_{2}: 0.25 \mathrm{R}_{1} \mathrm{OH}: 0.25 \mathrm{R}_{2} \mathrm{OH}: 0.2$ L-leucine : $0.7 \mathrm{HF}: y \mathrm{H} 2 \mathrm{O}$ ).

\begin{tabular}{cccccc}
$\#$ & $\boldsymbol{y}$ & $\mathbf{T}\left({ }^{\circ} \mathbf{C}\right)$ & Condition & Time (days) & Result \\
\hline R1R2-L2-175 & 4.0 & 175 & Rotating & 7 & STW \\
R1R2-L2-175* & 7.0 & 175 & Rotating & 7 & HPM-2
\end{tabular}


(a) $100 \% 12345 \mathrm{PMIOH}$ gel $\mathrm{R} 1-\mathrm{I} 2$

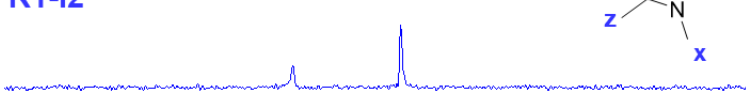

$50 \%$ 12345PMIOH + 50\% 2E134TMIOH gel

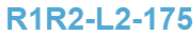

R1R2-L2-175
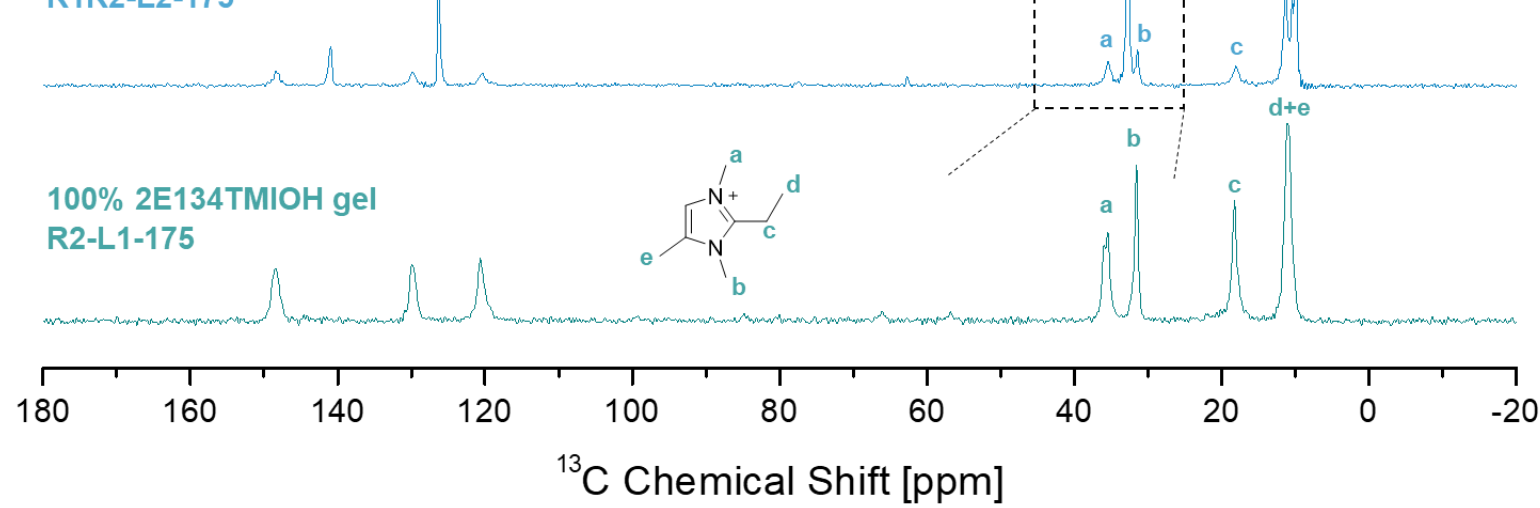

(b)

(b) 2x area: $73.63 \%$

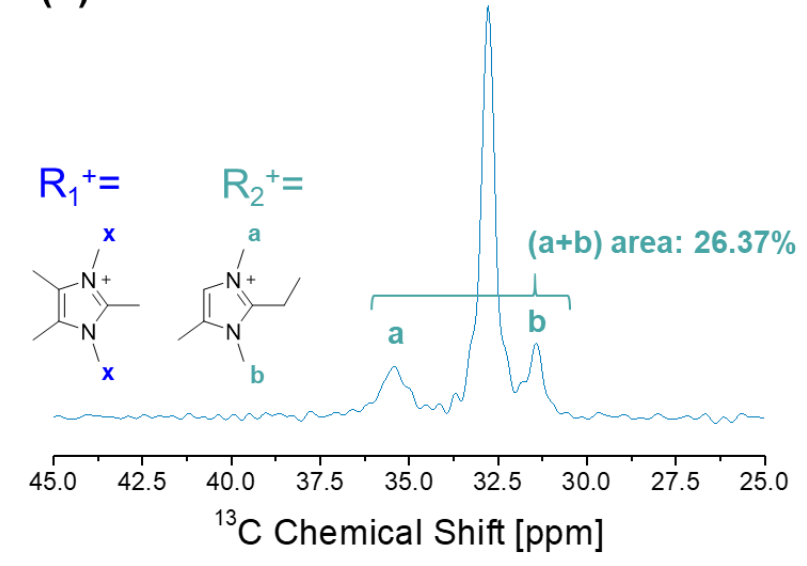

Figure S9. (a) ${ }^{1} \mathrm{H}-{ }^{13} \mathrm{C}$ CPMAS (8 kHz) NMR spectra of as-made pure-silica STW from $100 \%$ 12345PMI (top), the molar 1:1 mixture of 12345PMI and 2E134TMI (R1R2-L2-175, middle), and 100\% 2E134TMI (bottom). (b) Spectrum in the range of $\mathrm{N}^{-\mathrm{CH}_{3}}$ resonance $(25-45 \mathrm{ppm})$ of the two OSDAs within the asmade R1R2-L2-175 STW (the region marked with a dashed box in (a)). 


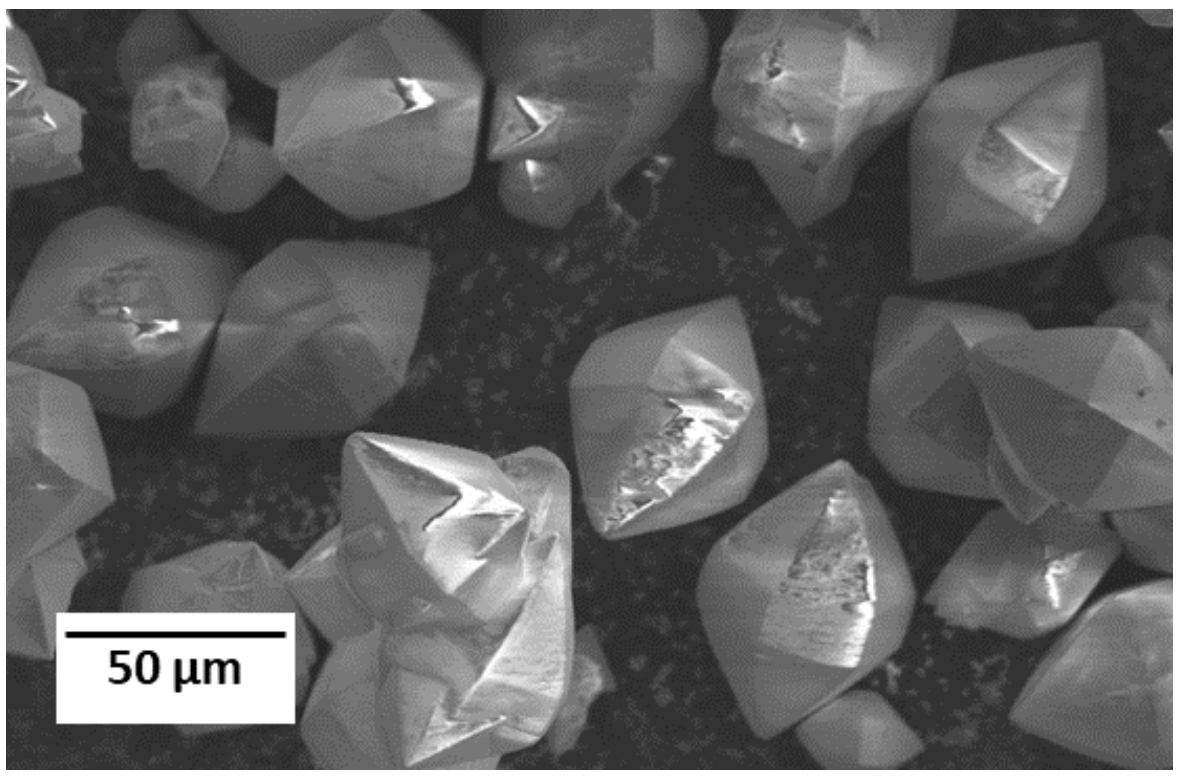

Figure S10. SEM image of as-made pure-silica STW R1R2-L2-175. 


\section{Crystallographic Information for Pure-silica STW Containing 12345PMI and 2E134TMI Monoquaternary OSDAs}

\section{Calcined Pure-silica STW (12345PMI)}

Table S6. Summary of crystallographic information of randomly selected 16 crystallites from the batch STW-R1-P2 obtained from the single-crystal XRD refinements.

\begin{tabular}{cccccc}
$\#$ & Space Group & $\mathbf{a}(\mathbf{A})$ & $\mathbf{c}(\mathbf{\AA})$ & Flack x & Hooft $\mathbf{~}$ \\
\hline 1 & $\mathrm{P} 6_{5} 22(\mathrm{R})$ & 11.934 & 29.831 & 0.05 & 0.03 \\
2 & $\mathrm{P} 6_{1} 22(\mathrm{~S})$ & 11.923 & 29.827 & -0.01 & -0.04 \\
3 & $\mathrm{P} 6_{1} 22(\mathrm{~S})$ & 11.929 & 29.834 & -0.08 & -0.1 \\
4 & $\mathrm{P} 6_{5} 22(\mathrm{R})$ & 11.924 & 29.785 & 0.05 & 0.01 \\
5 & $\mathrm{P} 6_{1} 22(\mathrm{~S})$ & 11.909 & 29.807 & 0.03 & 0 \\
6 & $\mathrm{P} 6_{5} 22(\mathrm{R})$ & 11.914 & 29.768 & -0.1 & -0.09 \\
7 & $\mathrm{P} 6_{5} 22(\mathrm{R})$ & 11.928 & 29.784 & 0.11 & 0.11 \\
8 & $\mathrm{P} 6_{1} 22(\mathrm{~S})$ & 11.864 & 29.718 & 0.21 & 0.18 \\
9 & $\mathrm{P} 6_{1} 22(\mathrm{~S})$ & 11.919 & 29.884 & 0.04 & 0.07 \\
10 & $\mathrm{P} 6_{1} 22(\mathrm{~S})$ & 11.927 & 29.814 & 0.03 & 0 \\
11 & $\mathrm{P} 6_{1} 22(\mathrm{~S})$ & 11.919 & 29.84 & 0.08 & 0.06 \\
12 & $\mathrm{P} 6_{5} 22(\mathrm{R})$ & 11.914 & 29.81 & -0.03 & -0.14 \\
13 & $\mathrm{P} 6_{5} 22(\mathrm{R})$ & 11.911 & 29.835 & 0.07 & 0.09 \\
14 & $\mathrm{P} 6_{5} 22(\mathrm{R})$ & 11.917 & 29.866 & 0.03 & 0.06 \\
15 & $\mathrm{P} 6_{5} 22(\mathrm{R})$ & 11.916 & 29.813 & 0.04 & 0 \\
16 & $\mathrm{P} 6_{5} 22(\mathrm{R})$ & 11.912 & 29.839 & 0.05 & 0.03 \\
\hline Average & & 11.916 & 29.816 & 0.04 & 0.02 \\
$($ St. Dev.) & & $(0.016)$ & $(0.039)$ & $(0.07)$ & $(0.08)$ \\
\hline
\end{tabular}



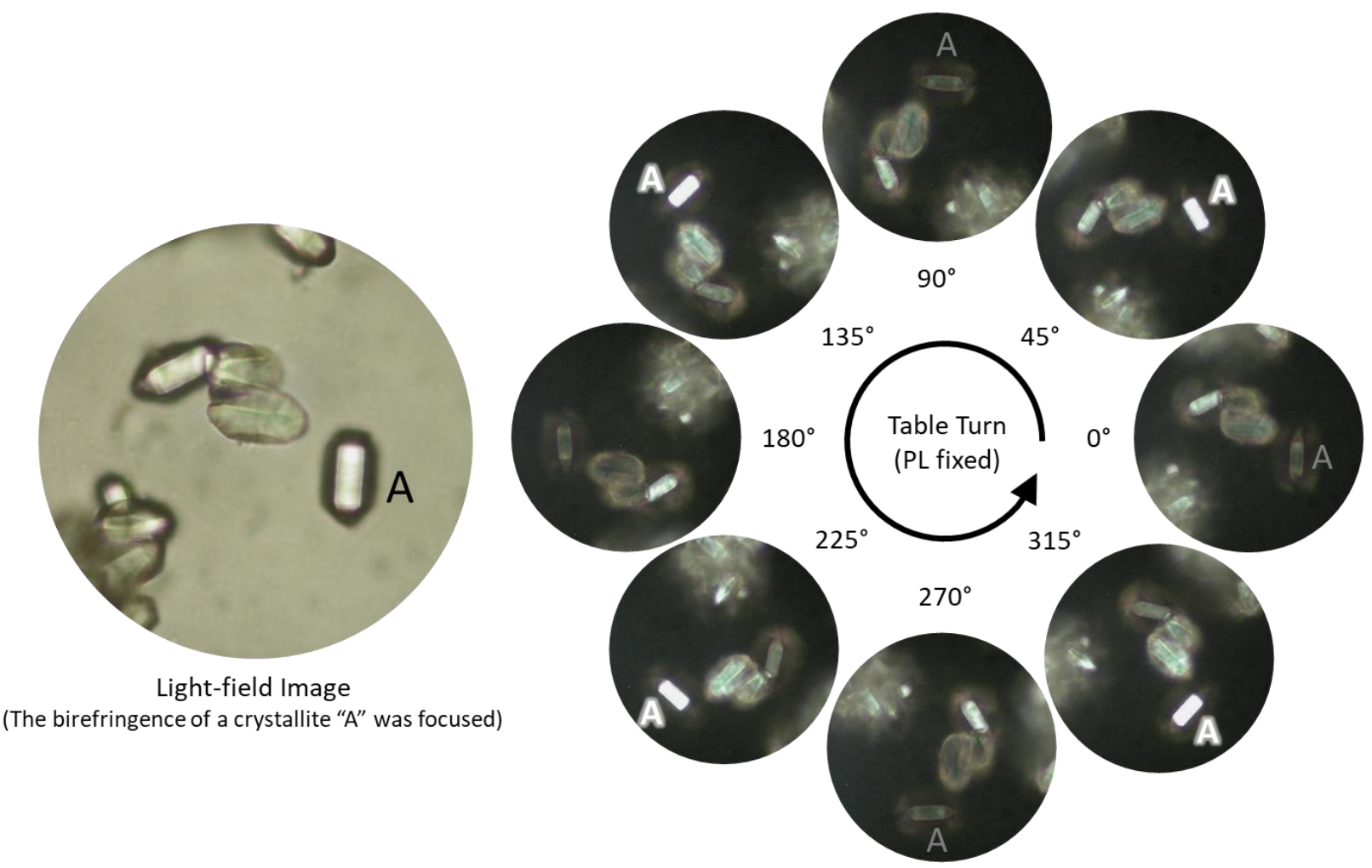

Dark-field Images

(" $A$ " glows every $90^{\circ}$ of rotation)

Figure S11. Optical and polarized optical microscopic images of calcined pure-silica STW-R1-P2. 
(a)

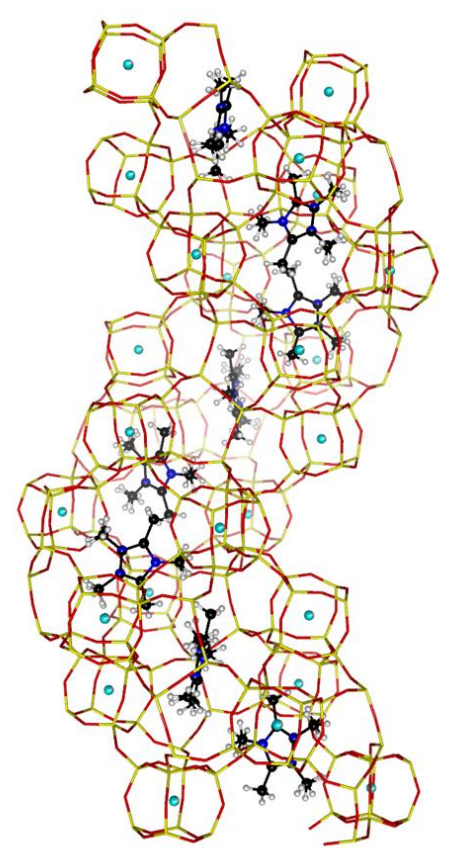

\section{Side-view \\ (projection along the $b$ axis)}

Top-view (projection along the $c$ axis) (b)
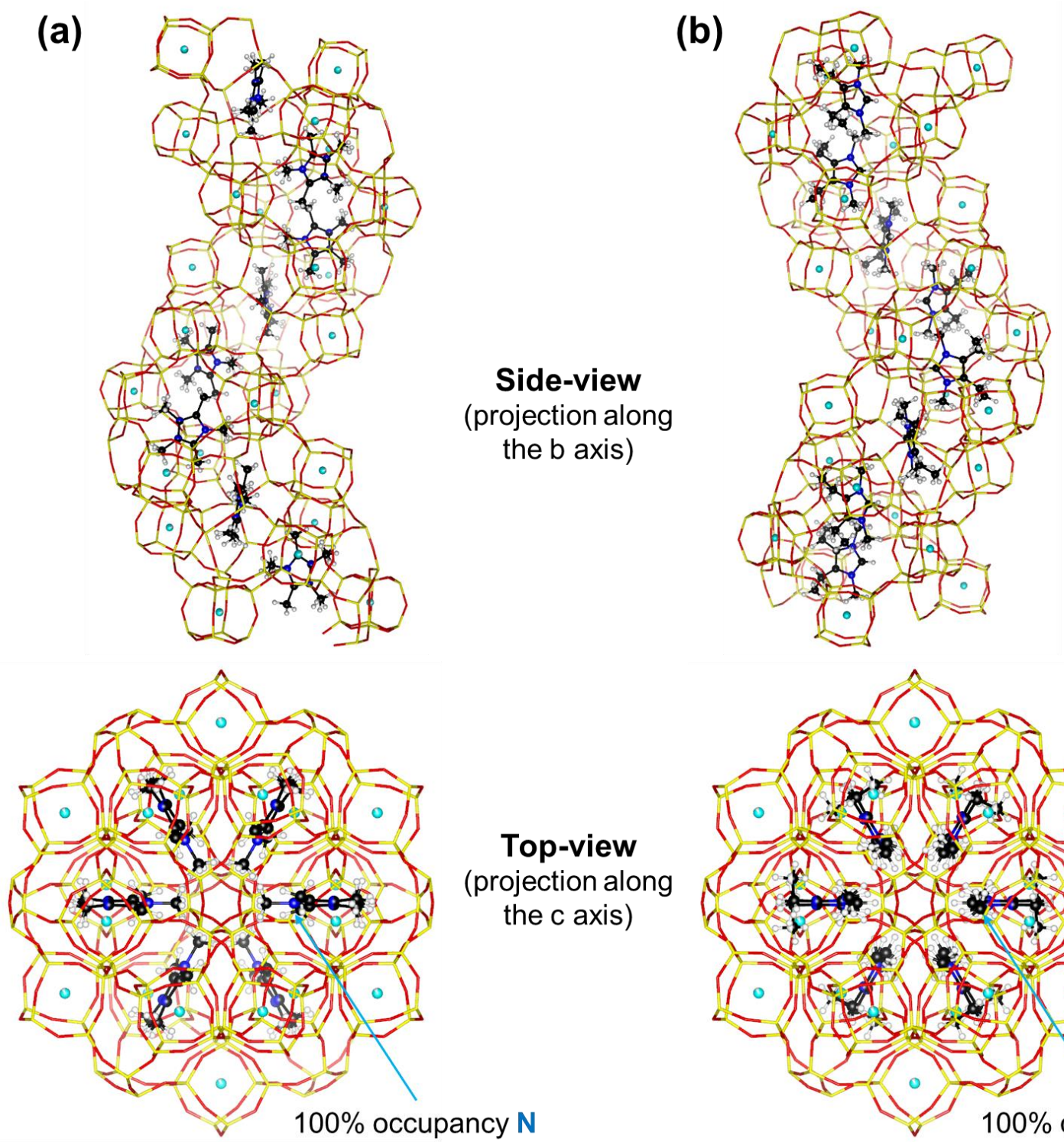

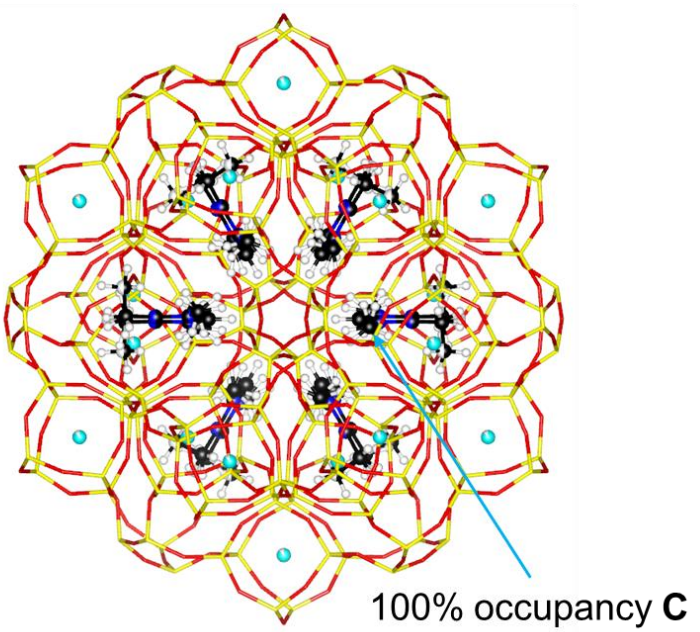

Figure S12. Projections (along the c axis) of helical channels of as-made pure-silica STW containing (a) 12345PMI and (b) 2E134TMI. 
Table S7. Crystallographic data for an as-made pure-silica 12345PMI R-STW crystallite determined based on the single-crystal X-ray diffraction. (Sample: R1-P2) (Excerpted from the full-version CIF file that is uploaded as a separate file in the Supporting Information available online.)

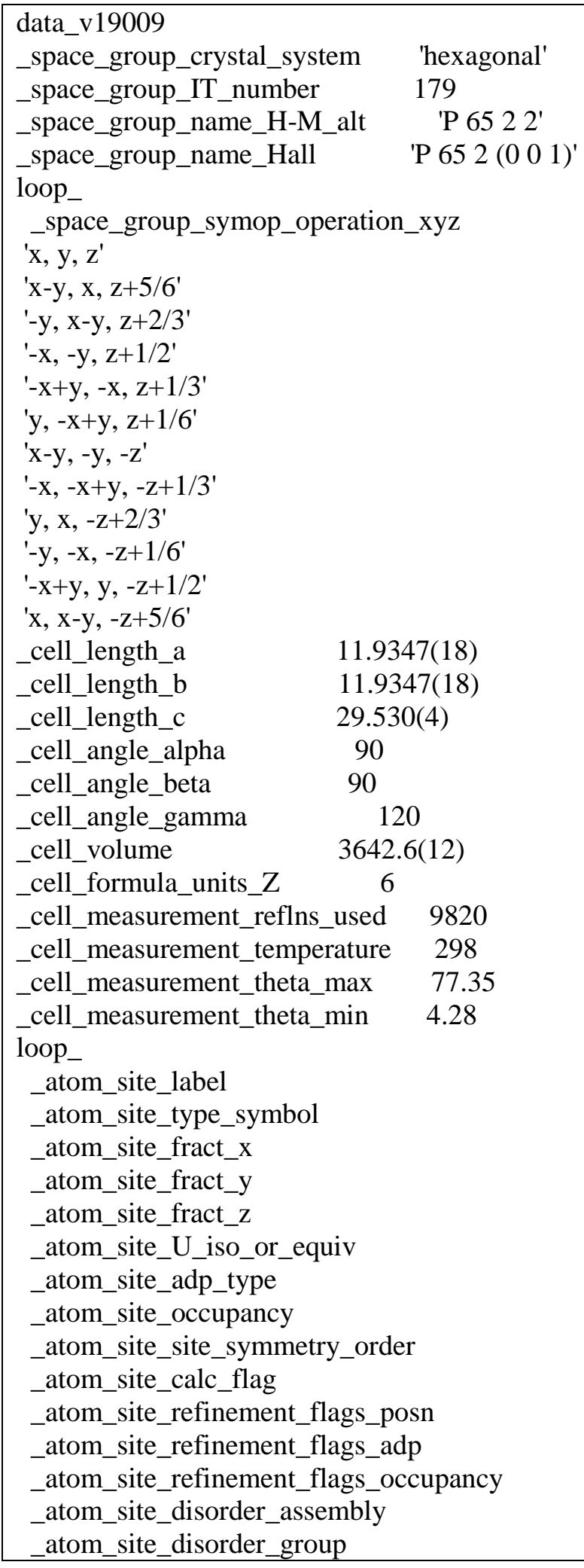




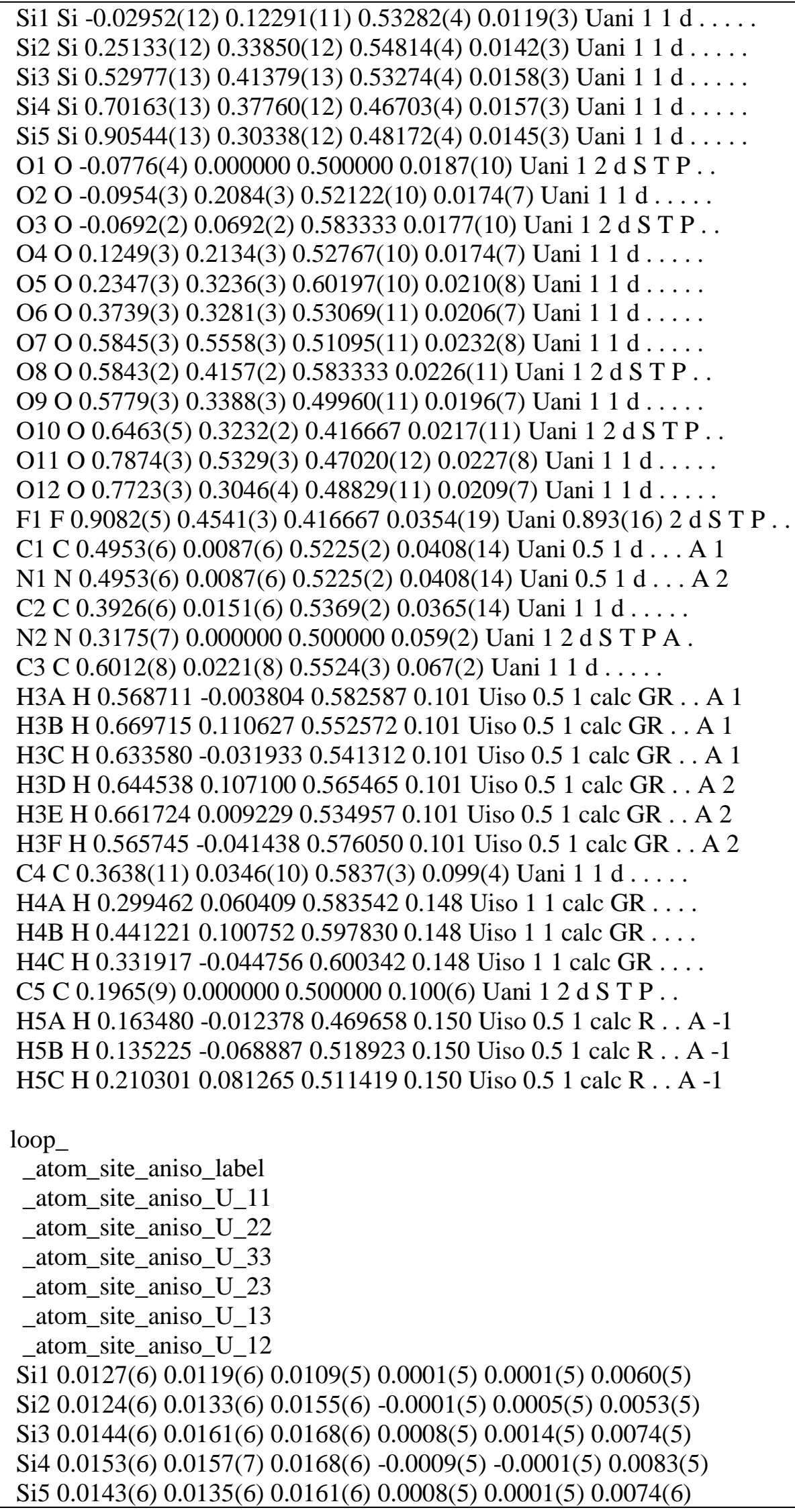




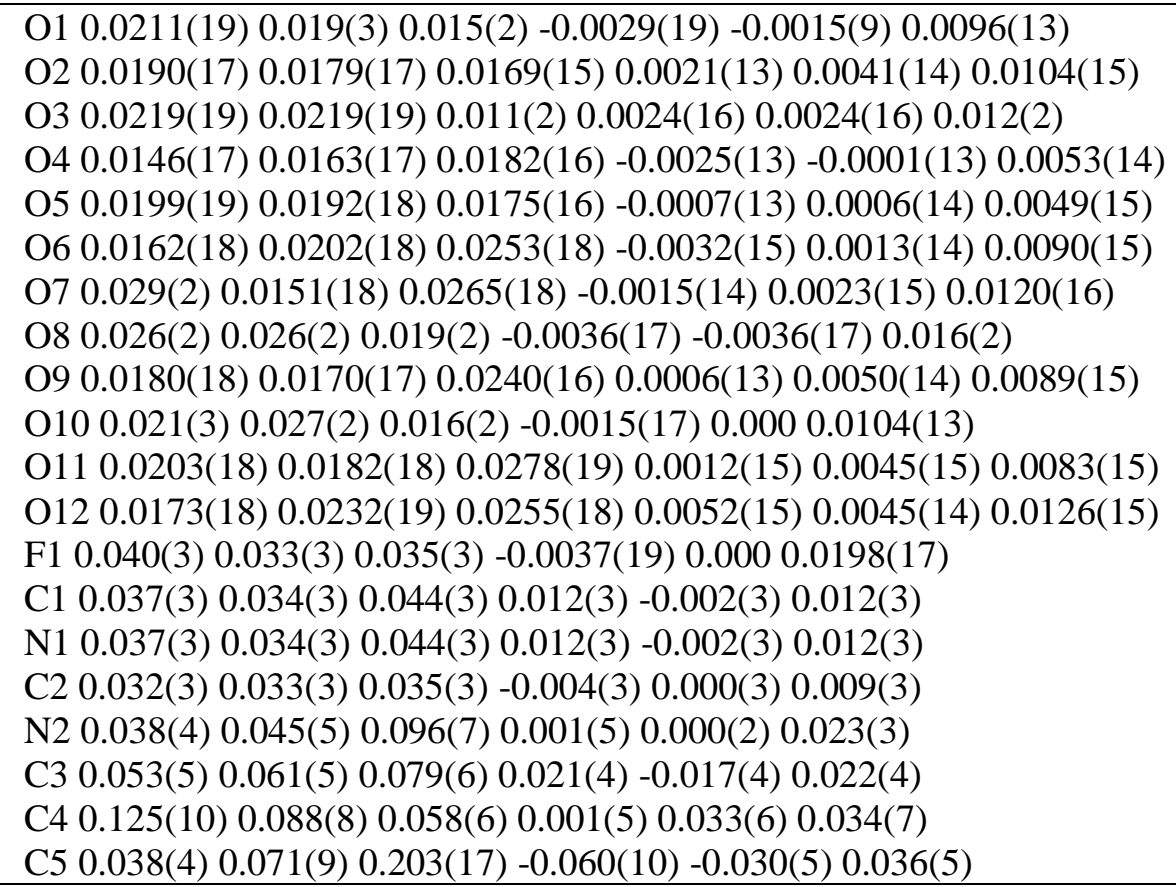


Table S8. Crystallographic data for an as-made pure-silica 2E134TMI S-STW crystallite based on the single-crystal X-ray diffraction. (Sample: R2-I1) (Excerpted from the full-version CIF file that is uploaded as a separate file in the Supporting Information available online.)

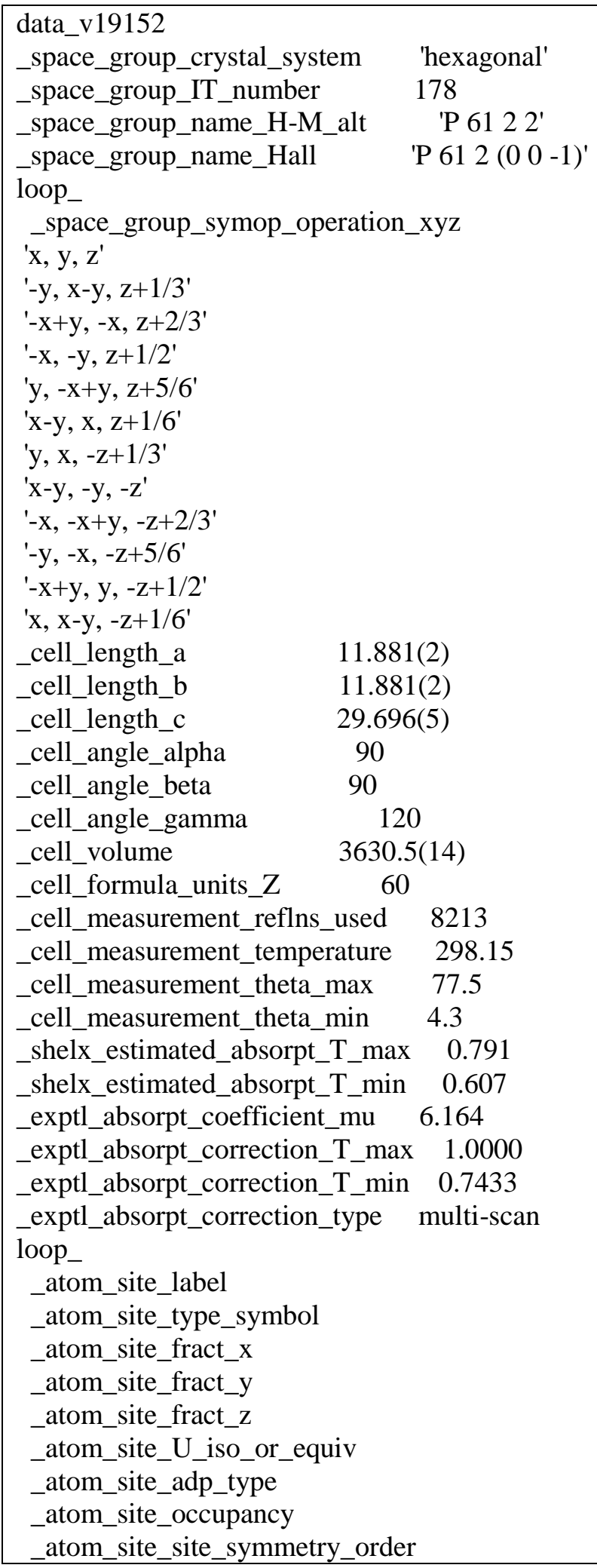




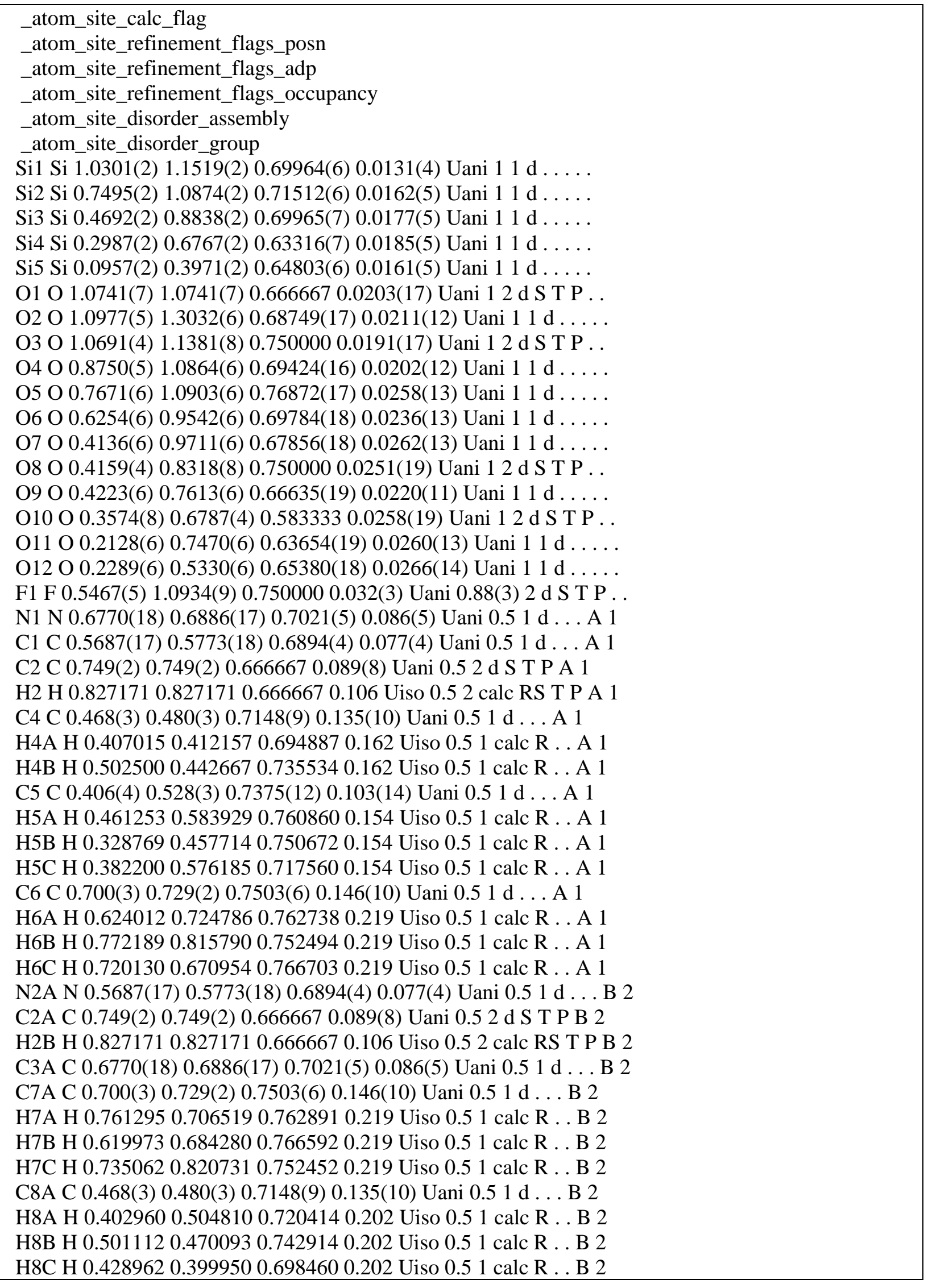




\begin{tabular}{|c|}
\hline $\begin{array}{l}\text { Oop_ } \\
\text { _atom_site_aniso_label } \\
\text { _atom_site_aniso_U_11 } \\
\text { _atom_site_aniso_U_22 } \\
\text { _atom_site_aniso_U_33 } \\
\text { _atom_site_aniso_U_23 } \\
\text { _atom_site_aniso_U_13 } \\
\text { _atom_site_aniso_U_12 } \\
\text { Si1 } 0.0126(10) 0.0150(11) 0.0138(8)-0.0007(7)-0.0003(7) 0.0085(9) \\
\text { Si2 } 0.0132(11) 0.0151(11) 0.0202(9)-0.0003(8)-0.0002(8) 0.0071(9) \\
\text { Si3 } 0.0164(11) 0.0137(11) 0.0238(10)-0.0008(8)-0.0012(8) 0.0080(9) \\
\text { Si4 } 0.0176(12) 0.0148(11) 0.0213(9)-0.0008(8)-0.0004(8) 0.0066(10) \\
\text { Si5 } 0.0159(11) 0.0124(11) 0.0205(9) 0.0008(8)-0.0002(8) 0.0074(9) \\
\text { O1 } 0.022(3) 0.022(3) 0.023(3)-0.0029(14) 0.0029(14) 0.016(4) \\
\text { O2 } 0.017(3) 0.018(3) 0.024(3) 0.003(2)-0.001(2) 0.005(3) \\
\text { O3 } 0.020(3) 0.023(4) 0.015(3) 0.000-0.001(2) 0.011(2) \\
\text { O4 } 0.020(3) 0.019(3) 0.021(3)-0.002(2)-0.001(2) 0.010(3) \\
\text { O5 } 0.021(3) 0.040(4) 0.024(3) 0.000(2) 0.001(2) 0.020(3) \\
\text { O6 } 0.017(3) 0.019(3) 0.034(3)-0.007(2)-0.002(2) 0.008(3) \\
\text { O7 } 0.028(4) 0.020(3) 0.036(3)-0.005(2)-0.007(3) 0.015(3) \\
\text { O8 } 0.024(4) 0.021(5) 0.029(4) 0.0000 .004(3) 0.011(2) \\
\text { O9 } 0.018(3) 0.016(3) 0.032(3)-0.005(2)-0.005(2) 0.009(3) \\
\text { O10 } 0.018(5) 0.030(4) 0.025(4)-0.001(3) 0.0000 .009(2) \\
\text { O11 } 0.022(3) 0.018(3) 0.037(3)-0.003(2)-0.005(2) 0.009(3) \\
\text { O12 } 0.025(3) 0.023(3) 0.026(3) 0.002(2)-0.004(2) 0.007(3) \\
\text { F1 } 0.023(4) 0.042(6) 0.039(5) 0.0000 .002(3) 0.021(3) \\
\text { N1 } 0.111(14) 0.089(12) 0.071(8) 0.009(8) 0.002(9) 0.059(12) \\
\text { C1 } 0.086(12) 0.085(12) 0.078(8) 0.014(8) 0.005(8) 0.056(10) \\
\text { C2 } 0.106(17) 0.106(17) 0.082(15) 0.005(6)-0.005(6) 0.073(18) \\
\text { C4 } 0.13(2) 0.14(2) 0.20(3)-0.022(19)-0.016(19) 0.12(2) \\
\text { C5 } 0.10(3) 0.06(2) 0.11(3) 0.04(2)-0.03(2) 0.02(2) \\
\text { C6 } 0.25(3) 0.17(2) 0.060(10)-0.022(12)-0.027(14) 0.14(2) \\
\text { N2A } 0.086(12) 0.085(12) 0.078(8) 0.014(8) 0.005(8) 0.056(10) \\
\text { C2A } 0.106(17) 0.106(17) 0.082(15) 0.005(6)-0.005(6) 0.073(18) \\
\text { C3A } 0.111(14) 0.089(12) 0.071(8) 0.009(8) 0.002(9) 0.059(12) \\
\text { C7A } 0.25(3) 0.17(2) 0.060(10)-0.022(12)-0.027(14) 0.14(2) \\
\text { C8A } 0.13(2) 0.14(2) 0.20(3)-0.022(19)-0.016(19) 0.12(2) \\
\end{array}$ \\
\hline
\end{tabular}



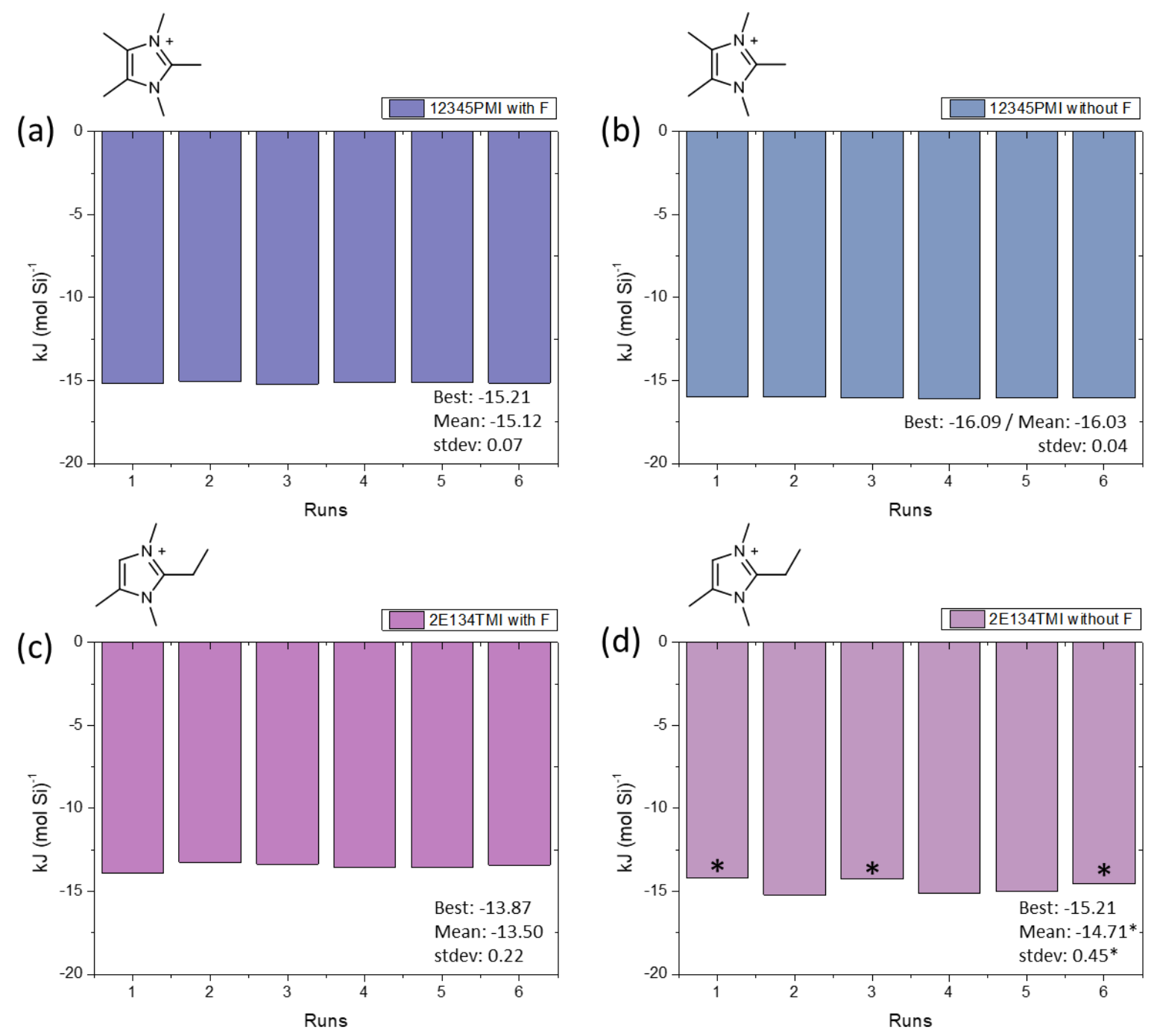

Figure S13. Wall-OSDA interaction energies calculated from the MD. Six independent MD runs are performed for each case: (a) 12345PMI with fluoride within d4r units, (b) 12345PMI without fluoride, (c) 2E134TMI with fluoride, and (d) 2E134TMI without fluoride. Asterisks (*) denote simulations with less optimal configurations and energies- highlighting the need for multiple runs. 


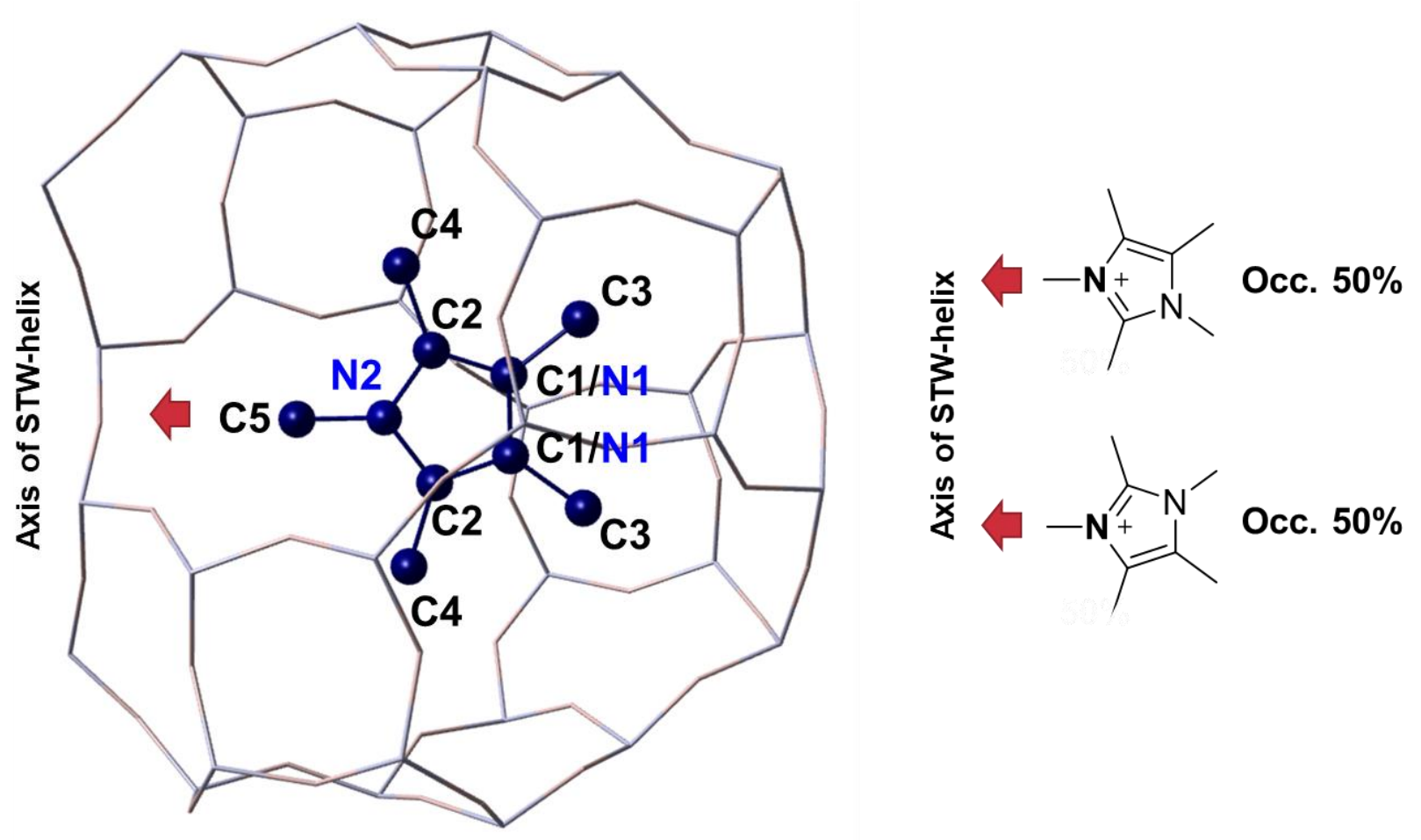

Figure S14. Visualization of an 12345PMI OSDA cation within a single cage of STW based on the structure solution from the single-crystal X-ray diffraction. Atom numberings are the same as the provided CIF file.

Table S9. Atomic displacement parameters of atoms of 12345PMI OSDA within an STW cage characterized based on the single-crystal X-ray diffraction experiment at room temperature (298 K). Atom numberings are the same as the provided CIF file. $\mathrm{L}^{1}(\mathrm{U})$ is the 1-norm (tensor magnitude) of each atomic displacement tensor that is defined as $\mathrm{L}^{1}(U)=\max _{1 \leq j \leq 3}\left(\sum_{j=1}^{3}\left|U_{i j}\right|\right)$.

\begin{tabular}{|c|c|c|c|c|c|c|c|c|}
\hline \multirow{2}{*}{ Atom } & \multirow{2}{*}{ Occupancy } & \multicolumn{6}{|c|}{ Atomic Displacement Tensor Elements $(\AA)$} & \multirow{2}{*}{$\mathrm{L}^{1}(\mathrm{U})$} \\
\cline { 2 - 8 } & & $\mathrm{U} 11$ & $\mathrm{U} 22$ & $\mathrm{U} 33$ & $\mathrm{U} 23$ & $\mathrm{U} 13$ & $\mathrm{U} 12$ & \\
\hline C1 (ring) & 0.5 & $0.037(3)$ & $0.034(3)$ & $0.044(3)$ & $0.012(3)$ & $-0.002(3)$ & $0.012(3)$ & 0.058 \\
\hline C2 (ring) & 1 & $0.032(3)$ & $0.033(3)$ & $0.035(3)$ & $-0.004(3)$ & $0.000(3)$ & $0.009(3)$ & 0.046 \\
\hline C3 (methyl) & 1 & $0.053(5)$ & $0.061(5)$ & $0.079(6)$ & $0.021(4)$ & $-0.017(4)$ & $0.022(4)$ & 0.117 \\
\hline C4 (methyl) & 1 & $0.125(10)$ & $0.088(8)$ & $0.058(6)$ & $0.001(5)$ & $0.033(6)$ & $0.034(7)$ & 0.192 \\
\hline C5 (methyl) & 1 & $0.038(4)$ & $0.071(9)$ & $0.203(17)$ & $-0.060(10)$ & $-0.030(5)$ & $0.036(5)$ & 0.293 \\
\hline N1 (ring) & 0.5 & $0.037(3)$ & $0.034(3)$ & $0.044(3)$ & $0.012(3)$ & $-0.002(3)$ & $0.012(3)$ & 0.058 \\
\hline N2 (ring) & 1 & $0.038(4)$ & $0.045(5)$ & $0.096(7)$ & $0.001(5)$ & $0.000(2)$ & $0.023(3)$ & 0.097 \\
\hline
\end{tabular}



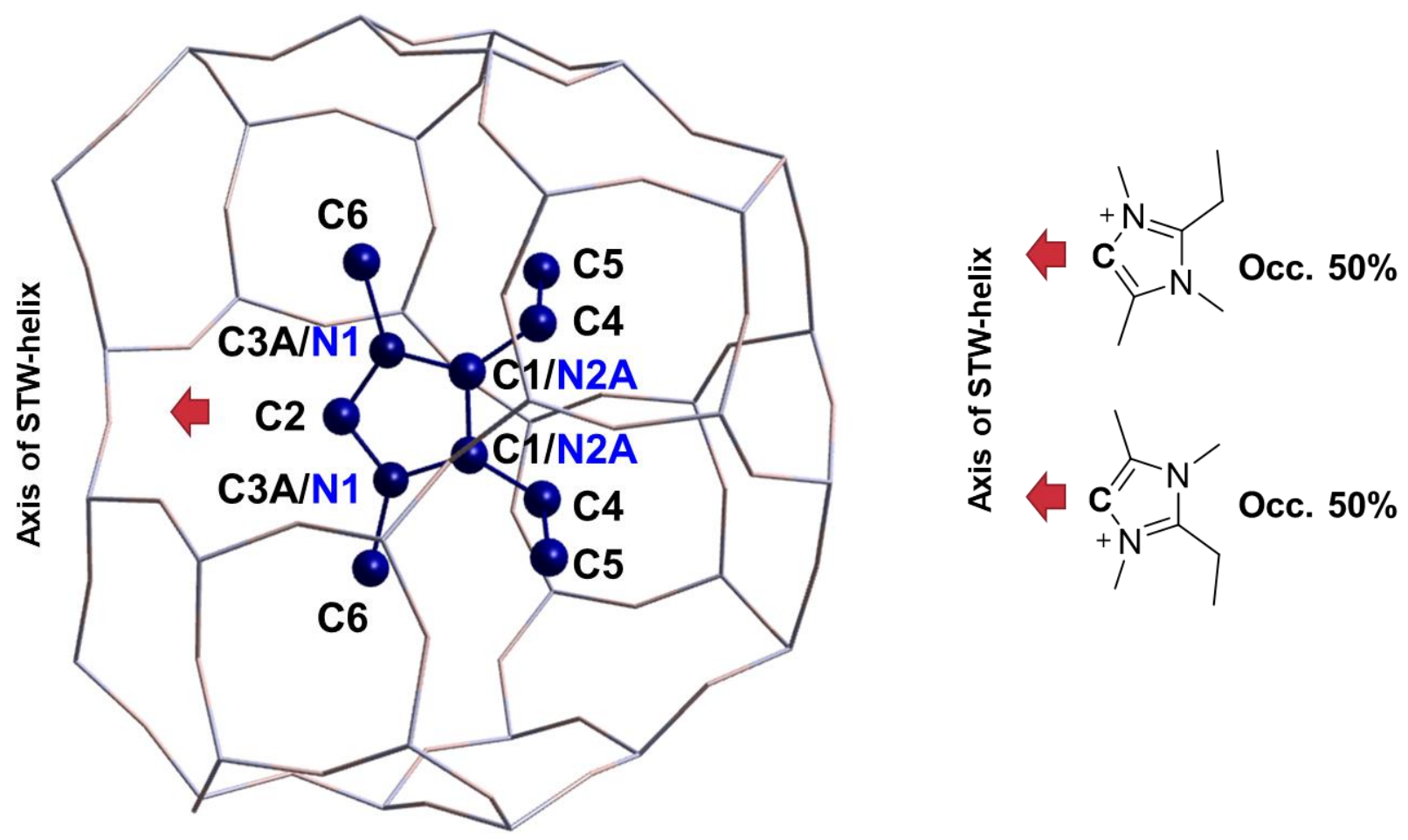

Figure S15. Visualization of a 2E134TMI OSDA cation within a single cage of STW based on the structure solution from the single-crystal X-ray diffraction. Atom numberings are the same as the provided CIF file.

Table S10. Atomic displacement parameters of atoms of 2E134TMI OSDA within an STW cage characterized based on the single-crystal X-ray diffraction experiment at room temperature (298 K). Atom numberings are the same as the provided CIF file. $\mathrm{L}^{1}(\mathrm{U})$ is the 1-norm (tensor magnitude) of each atomic displacement tensor that is defined as $\mathrm{L}^{1}(U)=\max _{1 \leq j \leq 3}\left(\sum_{j=1}^{3}\left|U_{i j}\right|\right)$.

\begin{tabular}{|c|c|c|c|c|c|c|c|c|}
\hline \multirow{2}{*}{ Atom } & \multirow{2}{*}{ Occupancy } & \multicolumn{6}{|c|}{ Atomic Displacement Tensor Elements $(\AA)$} & \multirow{2}{*}{$\mathrm{L}^{1}(\mathrm{U})$} \\
\hline & & U11 & $\mathrm{U} 22$ & $\mathrm{U} 33$ & $\mathrm{U} 23$ & U13 & U12 & \\
\hline C1 (ring) & 0.5 & $0.086(12)$ & $0.085(12)$ & $0.078(8)$ & $0.014(8)$ & $0.005(8)$ & $0.056(10)$ & 0.155 \\
\hline C2 (ring) & 1 & $0.106(17)$ & $0.106(17)$ & $0.082(15)$ & $0.005(6)$ & $-0.005(6)$ & $0.073(18)$ & 0.184 \\
\hline C3A (ring) & 0.5 & $0.111(14)$ & $0.089(12)$ & $0.071(8)$ & $0.009(8)$ & $0.002(9)$ & $0.059(12)$ & 0.172 \\
\hline $\begin{array}{c}\mathrm{C} 4 \\
\text { (methyl/ethyl) }\end{array}$ & 1 & $0.13(2)$ & 2) & 0. & $22(19)$ & $-0.016(19)$ & $0.12(2)$ & 82 \\
\hline C5 (ethyl) & 0.5 & $0.10(3)$ & $0.06(2)$ & $0.11(3)$ & $0.04(2)$ & $-0.03(2)$ & $0.02(2)$ & 0.180 \\
\hline C6 (methyl) & 1 & $0.25(3)$ & $0.17(2)$ & $0.060(10)$ & $-0.022(12)$ & $-0.027(14)$ & $0.14(2)$ & 0.417 \\
\hline N1 (ring) & 0.5 & $0.111(14)$ & $0.089(12)$ & $0.071(8)$ & $0.009(8)$ & $0.002(9)$ & $0.059(12)$ & 0.172 \\
\hline N2A (ring) & 0.5 & $0.086(12)$ & $0.085(12)$ & $0.078(8)$ & $0.014(8)$ & $0.005(8)$ & $0.056(10)$ & 0.155 \\
\hline
\end{tabular}



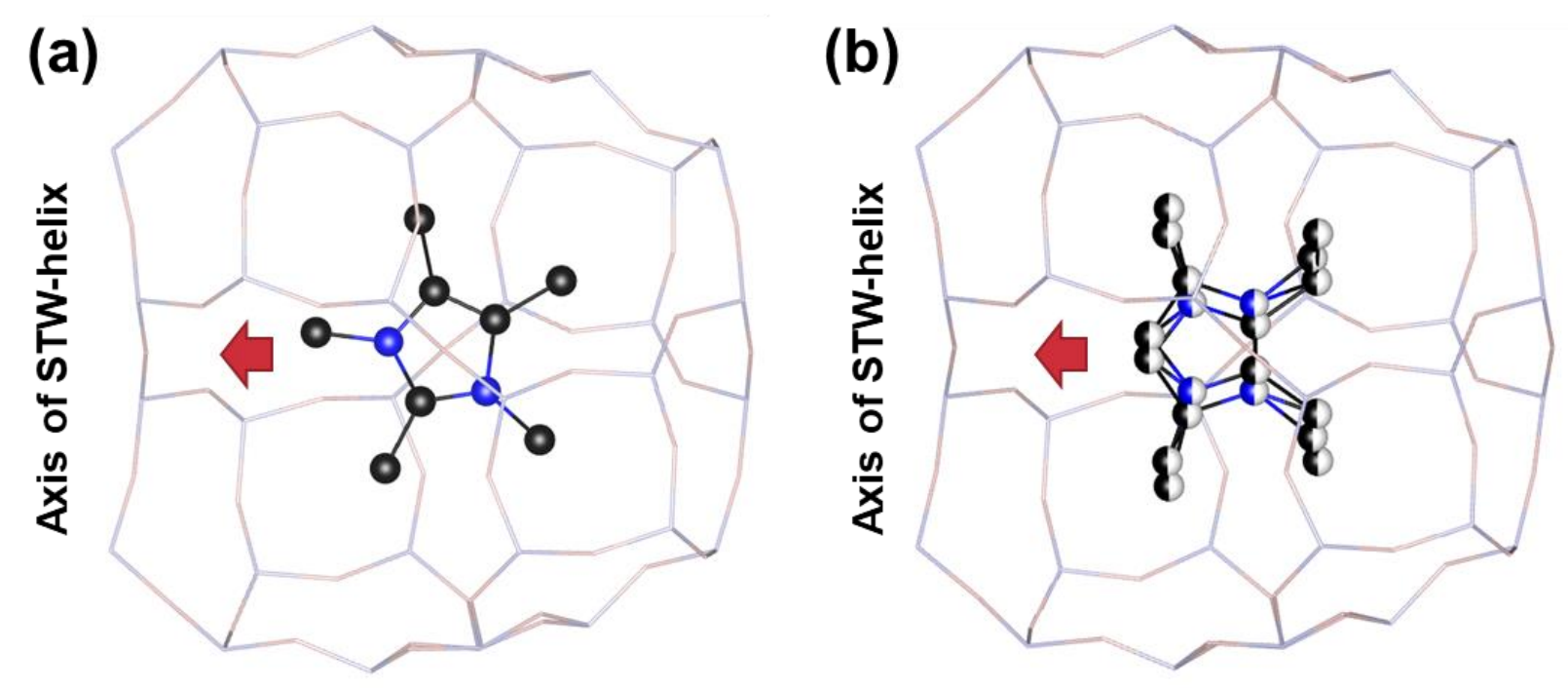

Figure S16. Visualizations of pure-silica STW cages with imidazolium-derived monoquaternary OSDAs: (a) 12345PMI and (b) 2E134TMI. Both (a) and (b) were reproduced from the CIF files obtained based on the Rietveld refinement of the synchrotron PXRD data by Yanhang Ma and Rojas et al. (2) The CIF file for (a) is by courtesy of Prof. Yanhang Ma at ShanghaiTech. The CIF file for (b) is available online as a part of the Supporting Information of Ref. (2). In the structure refined by Rojas et al., 2E134TMI cations occupy two crystallographically equivalent positions with a 50:50 occupancy ratio. 


\section{Synthesis and Characterization of Enantioenriched Germanosilicate R-STW Using Chiral Diquat OSDA}

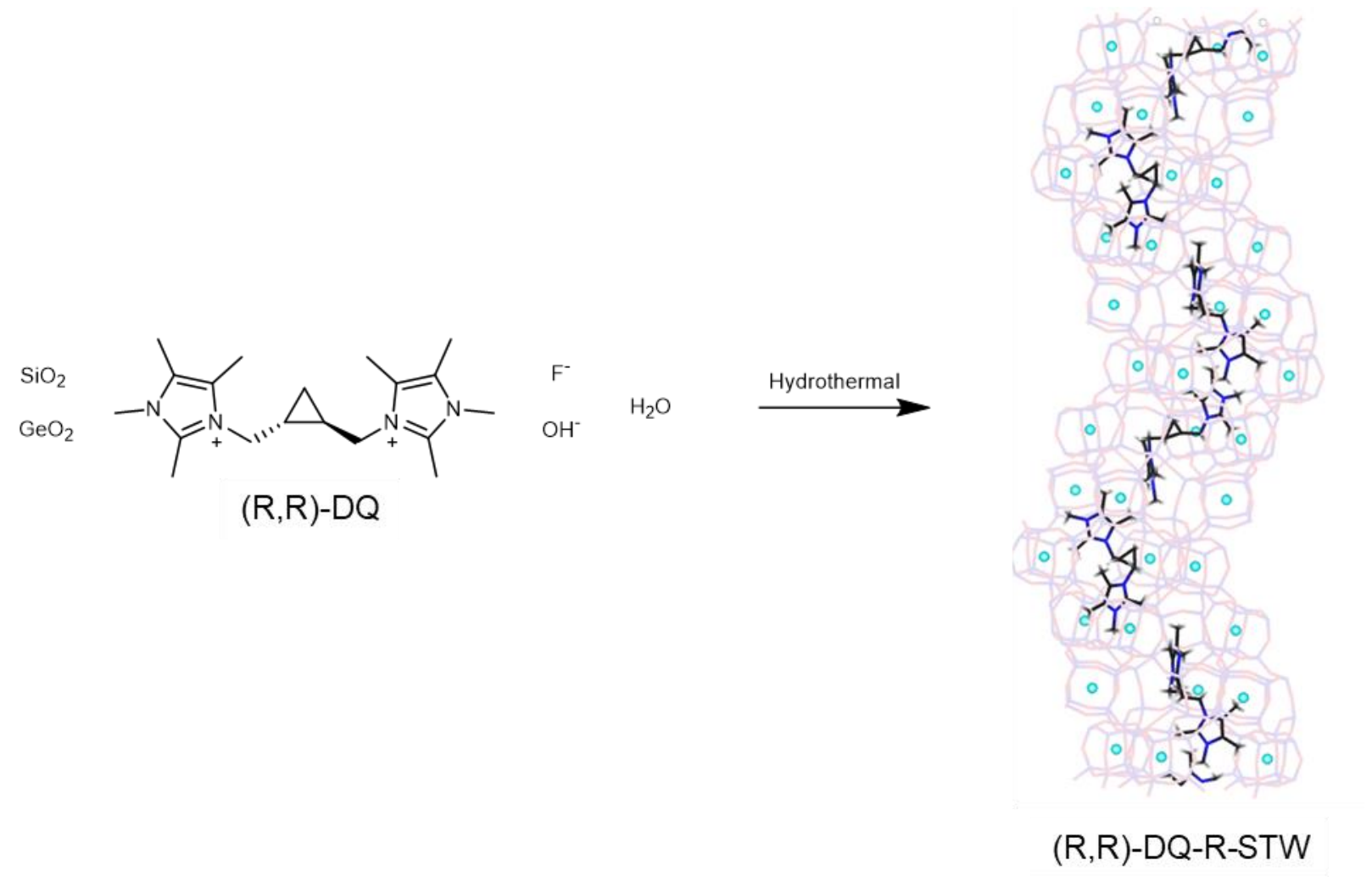

Figure S17. Schematic illustration of the synthesis of germanosilicate enantioenriched R-STW from (R,R)DQ OSDA.

\section{Synthesis Details (R-STW)}

Enantioenriched germanosilicate R-STW molecular sieve was synthesized using a chiral diquaternary OSDA, 3,3'-(((1R,2R)-cyclopropane-1,2-diyl)bis(methylene))bis(1,2,4,5-tetramethyl-1H-imidazol-3-ium) (denoted as (R,R)-DQ). (R,R)-DQ dihydroxide solution was prepared according to our previous report. (3) The gel composition formula was $1.0 \mathrm{SiO}_{2}: 0.5 \mathrm{GeO}_{2}: 0.5(\mathrm{R}, \mathrm{R})-\mathrm{DQ}(\mathrm{OH})_{2}: 0.5 \mathrm{HF}: 4.0 \mathrm{H}_{2} \mathrm{O}$. To be specific, $0.246 \mathrm{~g}(2.35 \mathrm{mmol})$ of germanium (IV) oxide (Strem Chemical, 99.999+\%) was fully dissolved in $5.07 \mathrm{~g}$ of $(\mathrm{R}, \mathrm{R})-\mathrm{DQ}(\mathrm{OH})_{2}$ solution $\left(0.464 \mathrm{mmol}(\mathrm{R}, \mathrm{R})-\mathrm{DQ} / \mathrm{g}\right.$ in $\left.\mathrm{H}_{2} \mathrm{O}\right)$ in a $23-\mathrm{mL}$ PTFE liner of Parr autoclave. $1.000 \mathrm{~g}(4.70 \mathrm{mmol})$ of tetraethyl orthosilicate (TEOS, Alfa Aesar, 99.9\%) was added to the mixture and hydrolyzed by stirring overnight. The access amount of water was evaporated under an airflow. After the gel became viscous, $0.098 \mathrm{~g}$ (2.35 mmol HF) of HF solution (Sigma-Aldrich, $48 \%$ in $\mathrm{H}_{2} \mathrm{O}$ ) was added and mechanically mixed using a PTFE rod. The resultant sticky gel was further dried overnight in a fume hood. Finally, a desired amount of distilled water was added to achieve the desired water content, and the gel was tightly sealed in a Parr steel autoclave and placed in a rotating oven at $160{ }^{\circ} \mathrm{C}$. The progress of crystallization was monitored by taking aliquots periodically. The finished batch was washed with distilled water and acetone, and dried at $100{ }^{\circ} \mathrm{C}$ overnight. 


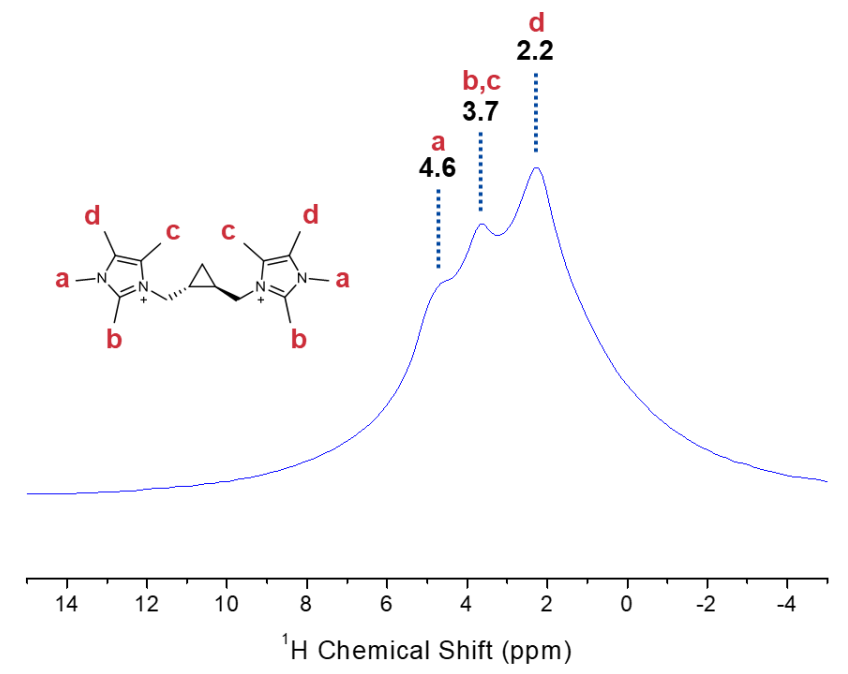

Figure S18. ${ }^{1} \mathrm{H}$ MAS ( $8 \mathrm{kHz}$ ) NMR spectrum of the as-made R-STW germanosilicate. Non-methyl protons are not clearly visible in the spectrum due to the signal broadening.

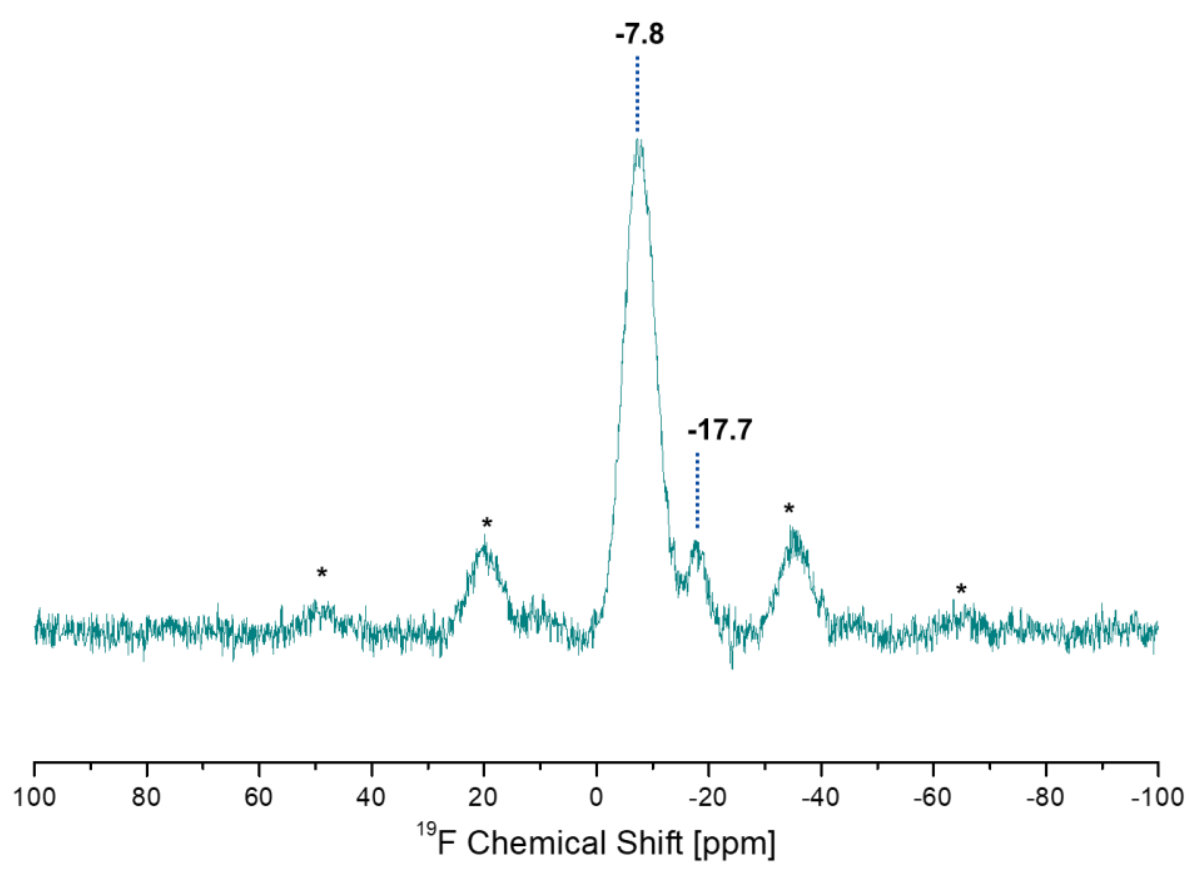

Figure S19. ${ }^{19}$ F MAS (13 kHz) NMR spectrum of the as-made R-STW germanosilicate. Spinning sidebands are denoted with asterisks $(*)$. 


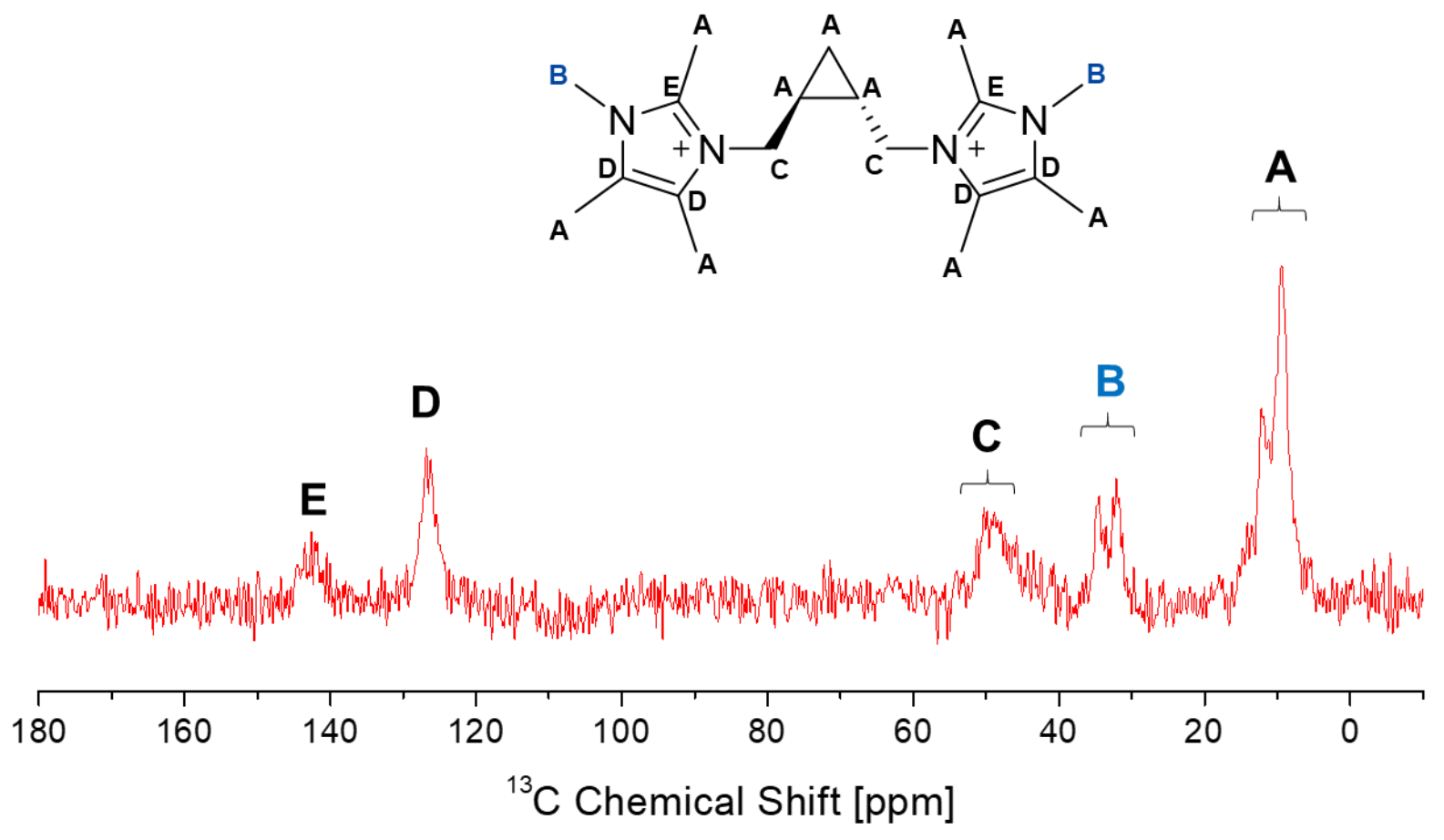

Figure S20. ${ }^{1} \mathrm{H}_{-}{ }^{13} \mathrm{C}$ CPMAS $(8 \mathrm{kHz})$ NMR spectrum of the as-made enantioenriched R-STW germanosilicate. The peak identification was guided by PerkinElmer ChemDraw Professional 17.0.0.206 Software. 


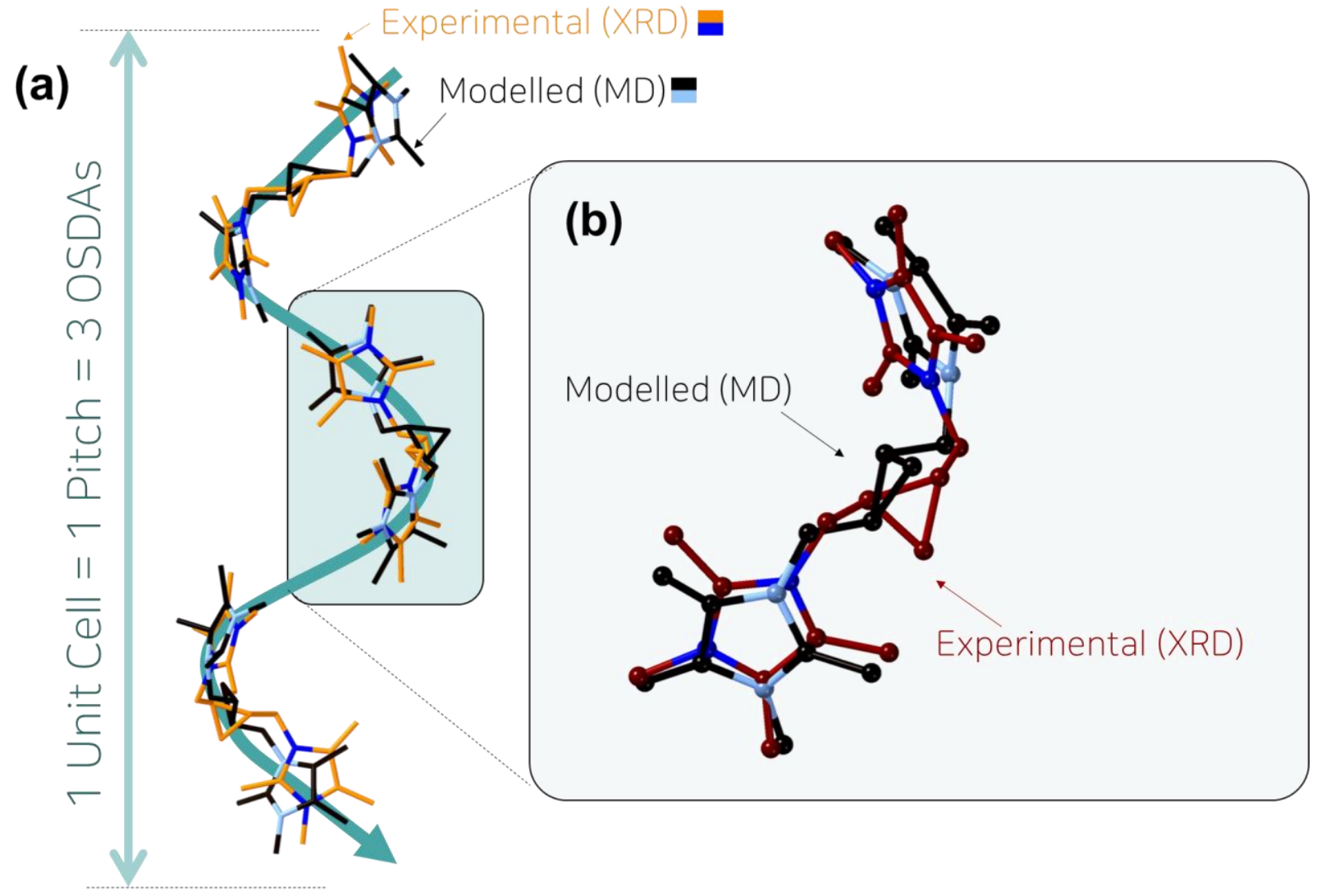

Figure S21. Visualizations of the modelled (C: black; N: light blue) and experimentally determined (C: brown/orange; $\mathrm{N}$ : blue) atomic positions of (a) three (R,R)-DQ OSDA dications within one unit cell and (b) one OSDA within an R-STW cage. 


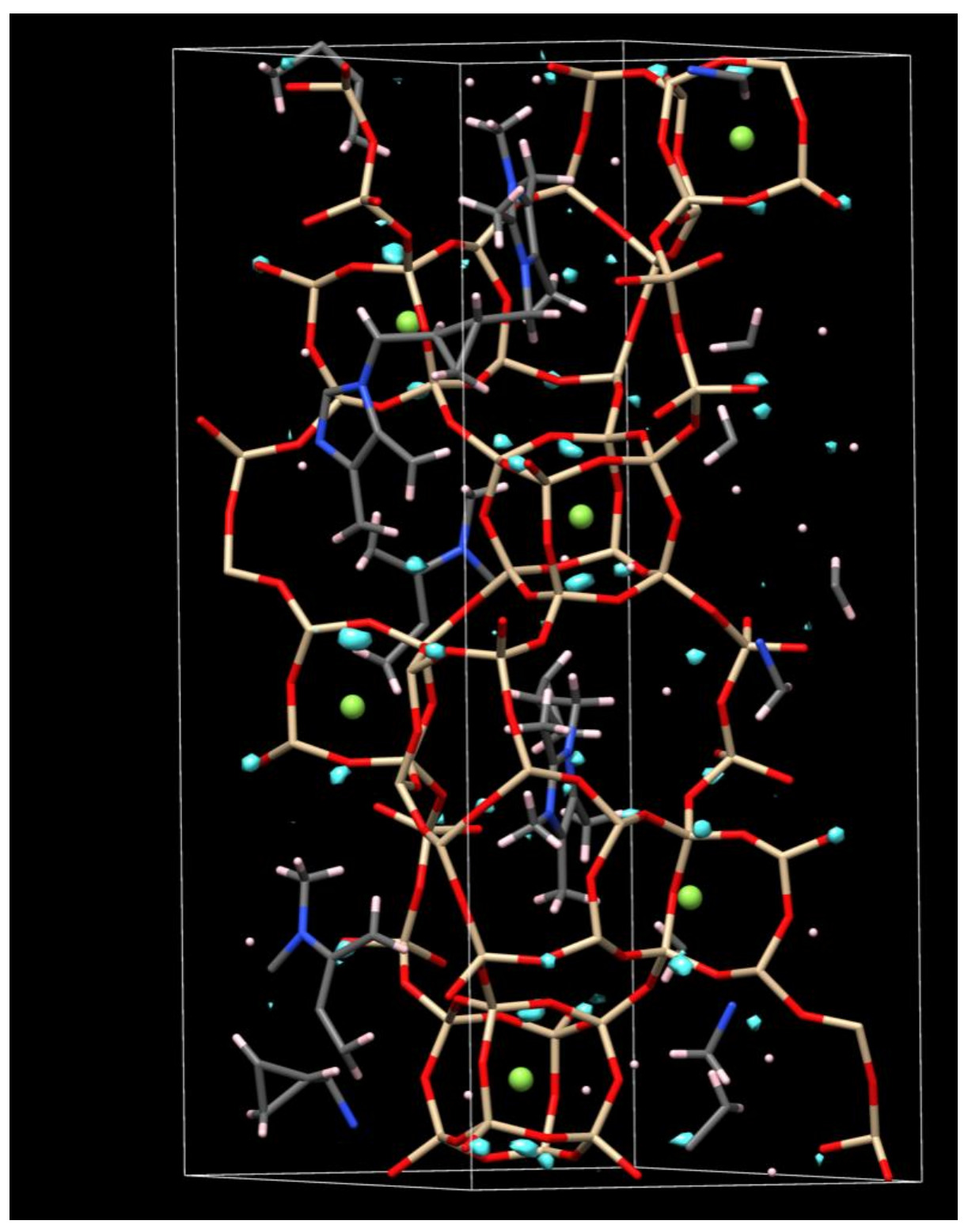

Figure S22. Visualization of a unit cell of as-made R-STW with the residual electron density (cyan colored) after the structure refinement. 


\section{Crystallographic Information for Germanosilicate R-STW}

Table S11. Crystallographic data for as-made R-STW acquired based on the synchrotron X-ray diffraction.

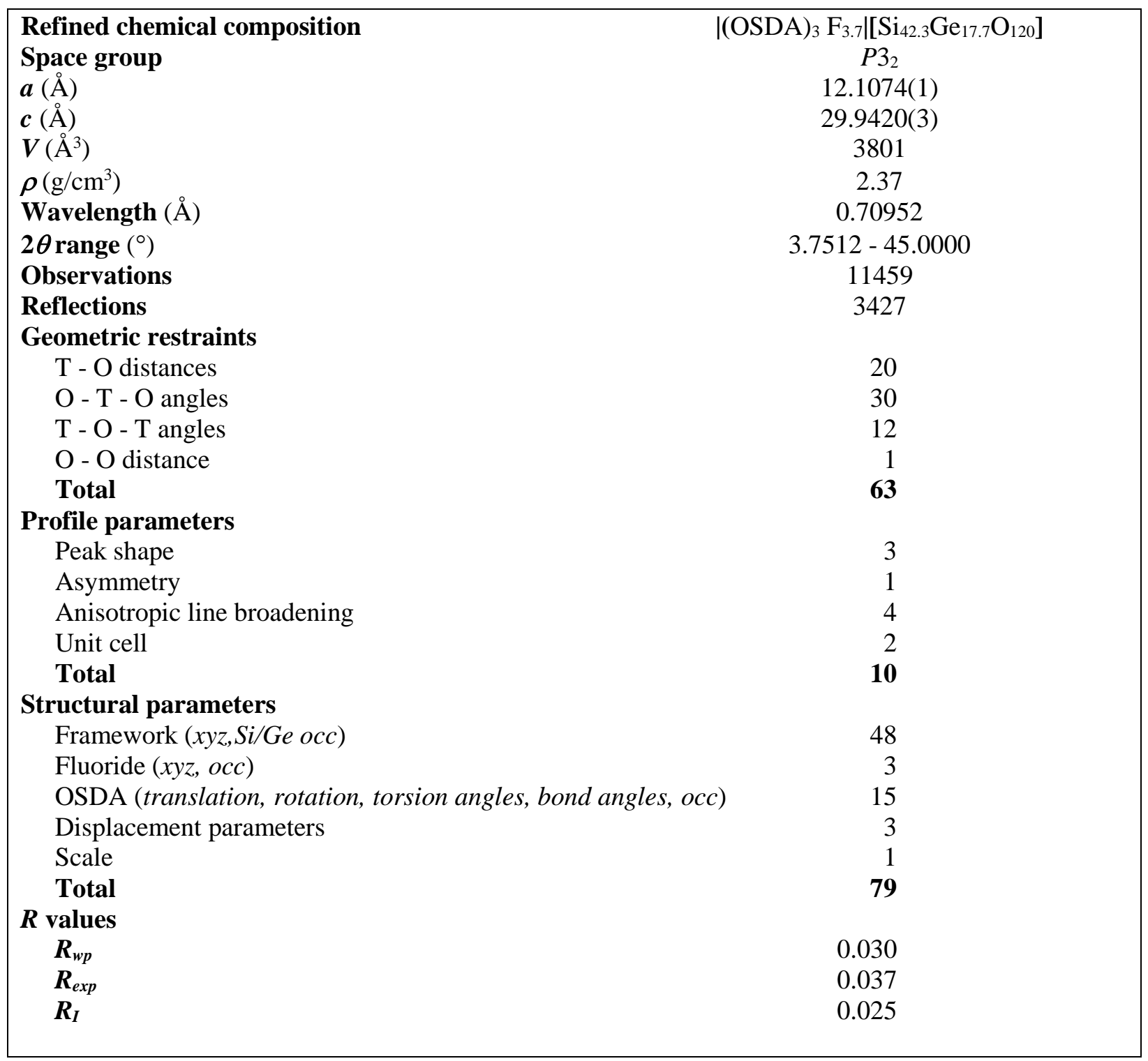


Table S12. Selected interatomic distances and angles for R-STW

\begin{tabular}{|c|c|c|c|}
\hline $\mathrm{T}(1)-\mathrm{O}(2)$ & $1.66(1)$ & $\mathrm{T}(4)-\mathrm{O}(3)$ & $1.62(1)$ \\
\hline $\mathrm{T}(1)-\mathrm{O}(6)$ & $1.67(1)$ & $\mathrm{T}(4)-\mathrm{O}(9)$ & $1.59(1)$ \\
\hline $\mathrm{T}(1)-\mathrm{O}(8)$ & $1.64(1)$ & $\mathrm{T}(4)-\mathrm{O}(76)$ & $1.61(1)$ \\
\hline $\mathrm{T}(1)-\mathrm{O}(9)$ & $1.63(1)$ & $\mathrm{T}(4)-\mathrm{O}(107)$ & $1.62(1)$ \\
\hline $\mathrm{T}(2)-\mathrm{O}(2)$ & $1.68(1)$ & $\mathrm{T}(5)-\mathrm{O}(1)$ & $1.63(1)$ \\
\hline $\mathrm{T}(2)-\mathrm{O}(7)$ & $1.65(1)$ & $\mathrm{T}(5)-\mathrm{O}(4)$ & $1.61(1)$ \\
\hline $\mathrm{T}(2)-\mathrm{O}(11)$ & $1.65(1)$ & $\mathrm{T}(5)-\mathrm{O}(5)$ & $1.59(1)$ \\
\hline $\mathrm{T}(2)-\mathrm{O}(12)$ & $1.65(1)$ & $\mathrm{T}(5)-\mathrm{O}(36)$ & $1.59(1)$ \\
\hline $\mathrm{T}(3)-\mathrm{O}(5)$ & $1.64(1)$ & & \\
\hline $\mathrm{T}(3)-\mathrm{O}(8)$ & $1.61(1)$ & & \\
\hline $\mathrm{T}(3)-\mathrm{O}(10)$ & $1.64(1)$ & & \\
\hline $\mathrm{T}(3)-\mathrm{O}(116)$ & $1.60(1)$ & & \\
\hline $\mathrm{O}(2)-\mathrm{T}(1)-\mathrm{O}(6)$ & $106.6(5)$ & $\mathrm{O}(3)-\mathrm{T}(4)-\mathrm{O}(9)$ & $110.3(5)$ \\
\hline $\mathrm{O}(2)-\mathrm{T}(1)-\mathrm{O}(8)$ & $104.6(5)$ & $\mathrm{O}(3)-\mathrm{T}(4)-\mathrm{O}(76)$ & $110.0(5)$ \\
\hline $\mathrm{O}(2)-\mathrm{T}(1)-\mathrm{O}(9)$ & $110.3(5)$ & $\mathrm{O}(3)-\mathrm{T}(4)-\mathrm{O}(107)$ & $104.6(4)$ \\
\hline $\mathrm{O}(6)-\mathrm{T}(1)-\mathrm{O}(8)$ & $110.8(3)$ & $\mathrm{O}(9)-\mathrm{T}(4)-\mathrm{O}(76)$ & $110.5(5)$ \\
\hline $\mathrm{O}(6)-\mathrm{T}(1)-\mathrm{O}(9)$ & $113.8(4)$ & $\mathrm{O}(9)-\mathrm{T}(4)-\mathrm{O}(107)$ & $110.9(5)$ \\
\hline $\mathrm{O}(8)-\mathrm{T}(1)-\mathrm{O}(9)$ & $110.3(5)$ & $\mathrm{O}(76)-\mathrm{T}(4)-\mathrm{O}(107)$ & $110.4(5)$ \\
\hline $\mathrm{O}(2)-\mathrm{T}(2)-\mathrm{O}(7)$ & $105.3(5)$ & $\mathrm{O}(1)-\mathrm{T}(5)-\mathrm{O}(4)$ & $107.5(3)$ \\
\hline $\mathrm{O}(2)-\mathrm{T}(2)-\mathrm{O}(11)$ & $109.6(5)$ & $\mathrm{O}(1)-\mathrm{T}(5)-\mathrm{O}(5)$ & $113.9(5)$ \\
\hline $\mathrm{O}(2)-\mathrm{T}(2)-\mathrm{O}(12)$ & $105.5(5)$ & $\mathrm{O}(1)-\mathrm{T}(5)-\mathrm{O}(36)$ & $111.9(5)$ \\
\hline $\mathrm{O}(7)-\mathrm{T}(2)-\mathrm{O}(11)$ & $112.3(5)$ & $\mathrm{O}(4)-\mathrm{T}(5)-\mathrm{O}(5)$ & $108.8(5)$ \\
\hline $\mathrm{O}(7)-\mathrm{T}(2)-\mathrm{O}(12)$ & 109.9(3) & $\mathrm{O}(4)-\mathrm{T}(5)-\mathrm{O}(36)$ & $110.8(4)$ \\
\hline $\mathrm{O}(11)-\mathrm{T}(2)-\mathrm{O}(12)$ & $113.6(4)$ & $\mathrm{O}(5)-\mathrm{T}(5)-\mathrm{O}(36)$ & $104.0(6)$ \\
\hline $\mathrm{O}(5)-\mathrm{T}(3)-\mathrm{O}(8)$ & $109.9(5)$ & & \\
\hline $\mathrm{O}(5)-\mathrm{T}(3)-\mathrm{O}(10)$ & $106.5(4)$ & & \\
\hline $\mathrm{O}(5)-\mathrm{T}(3)-\mathrm{O}(116)$ & $110.1(5)$ & & \\
\hline $\mathrm{O}(8)-\mathrm{T}(3)-\mathrm{O}(10)$ & $111.8(5)$ & & \\
\hline $\mathrm{O}(8)-\mathrm{T}(3)-\mathrm{O}(116)$ & $107.1(6)$ & & \\
\hline $\mathrm{O}(10)-\mathrm{T}(3)-\mathrm{O}(116)$ & $111.6(5)$ & & \\
\hline $\mathrm{T}(5)-\mathrm{O}(1)-\mathrm{T}(53)$ & $170.2(6)$ & $\mathrm{T}(2)-\mathrm{O}(7)-\mathrm{T}(43)$ & $140.6(5)$ \\
\hline $\mathrm{T}(1)-\mathrm{O}(2)-\mathrm{T}(2)$ & $133.3(6)$ & $\mathrm{T}(1)-\mathrm{O}(8)-\mathrm{T}(3)$ & $141.3(6)$ \\
\hline $\mathrm{T}(4)-\mathrm{O}(3)-\mathrm{T}(53)$ & $148.8(7)$ & $\mathrm{T}(1)-\mathrm{O}(9)-\mathrm{T}(4)$ & $151.1(6)$ \\
\hline $\mathrm{T}(5)-\mathrm{O}(4)-\mathrm{T}(54)$ & $168.6(3)$ & $\mathrm{T}(3)-\mathrm{O}(10)-\mathrm{T}(44)$ & $143.9(4)$ \\
\hline $\mathrm{T}(3)-\mathrm{O}(5)-\mathrm{T}(5)$ & $141.6(6)$ & $\mathrm{T}(2)-\mathrm{O}(11)-\mathrm{T}(33)$ & $152.6(6)$ \\
\hline $\mathrm{T}(1)-\mathrm{O}(6)-\mathrm{T}(14)$ & $139.6(8)$ & $\mathrm{T}(2)-\mathrm{O}(12)-\mathrm{T}(24)$ & $135.6(8)$ \\
\hline \multicolumn{4}{|c|}{ closest approach of OSDA to framework: } \\
\hline $\mathrm{C}(5)-\mathrm{O}(5)$ & $3.2(2)$ & $\mathrm{C}(19)-\mathrm{O}(57)$ & $3.2(3)$ \\
\hline $\mathrm{C}(5)-\mathrm{O}(8)$ & $3.1(2)$ & $\mathrm{C}(19)-\mathrm{O}(85)$ & $3.2(3)$ \\
\hline $\mathrm{C}(9)-\mathrm{O}(36)$ & $3.1(2)$ & $\mathrm{C}(23)-\mathrm{O}(35)$ & $3.1(1)$ \\
\hline $\mathrm{C}(12)-\mathrm{O}(35)$ & $3.1(3)$ & $\mathrm{C}(23)-\mathrm{O}(77)$ & $3.1(1)$ \\
\hline
\end{tabular}


Table S13. Coordinates, occupancies, and displacement parameters for the atoms in R-STW ${ }^{\mathrm{a}}$.

\begin{tabular}{|c|c|c|c|c|c|}
\hline Atom & $x$ & $y$ & $z$ & $o c c$ & $B$ \\
\hline Si1 & $0.3798(2)$ & $0.6759(2)$ & $0.1343(1)$ & $0.565(5)$ & $0.99(1)$ \\
\hline $\mathrm{Si} 2$ & $0.4134(2)$ & $0.8835(2)$ & $0.2003(1)$ & $0.594(4)$ & $0.99(1)$ \\
\hline $\mathrm{Si} 3$ & $0.2997(3)$ & $0.3953(3)$ & $0.1496(1)$ & $0.724(5)$ & $0.99(1)$ \\
\hline $\mathrm{Si} 4$ & $0.6628(2)$ & $0.7500(3)$ & $0.1181(1)$ & $0.704(5)$ & $0.99(1)$ \\
\hline $\mathrm{Si} 5$ & $0.1218(3)$ & $0.1503(3)$ & $0.1999(1)$ & $0.943(4)$ & $0.99(1)$ \\
\hline Ge1 & 0.3798 & 0.6759 & 0.1343 & 0.435 & $0.99(1)$ \\
\hline $\mathrm{Ge} 2$ & 0.4134 & 0.8835 & 0.2003 & 0.406 & $0.99(1)$ \\
\hline Ge3 & 0.2997 & 0.3953 & 0.1496 & 0.276 & $0.99(1)$ \\
\hline Ge4 & 0.6628 & 0.7500 & 0.1181 & 0.296 & $0.99(1)$ \\
\hline Ge5 & 0.1218 & 0.1503 & 0.1999 & 0.057 & $0.99(1)$ \\
\hline O1 & $00.0780(9)$ & 0.1667 & 1 & $2.01(5)$ & \\
\hline $\mathrm{O} 2$ & $0.3344(8)$ & $0.7570(9)$ & $0.1667(3)$ & 1 & $2.01(5)$ \\
\hline $\mathrm{O} 3$ & $0.7847(7)$ & $0.8785(8)$ & $0.1373(2)$ & 1 & $2.01(5)$ \\
\hline $\mathrm{O} 4$ & $0.0675(5)$ & 0.1350 & 0.25 & 1 & $2.01(5)$ \\
\hline O5 & $0.2104(7)$ & $0.2977(8)$ & 0.1894(3) & 1 & $2.01(5)$ \\
\hline O6 & $0.3245(5)$ & 0.6755 & 0.0833 & 1 & $2.01(5)$ \\
\hline $\mathrm{O} 7$ & $0.3272(8)$ & $0.9539(8)$ & $0.2012(2)$ & 1 & $2.01(5)$ \\
\hline O8 & $0.3087(8)$ & $0.5314(8)$ & $0.1555(3)$ & 1 & $2.01(5)$ \\
\hline O9 & $0.5340(8)$ & $0.7380(8)$ & $0.1364(3)$ & 1 & $2.01(5)$ \\
\hline $\mathrm{O} 10$ & $0.2321(7)$ & $0.3273(8)$ & $0.1021(3)$ & 1 & $2.01(5)$ \\
\hline O11 & $0.5584(8)$ & $0.9774(8)$ & $0.1802(3)$ & 1 & $2.01(5)$ \\
\hline $\mathrm{O} 12$ & $0.4121(5)$ & 0.8242 & 0.25 & 1 & $2.01(5)$ \\
\hline $\mathrm{F} 1$ & $0.9105(13)$ & $0.4553(7)$ & 0.91667 & $0.624(8)$ & $3.0(2)$ \\
\hline N1 & 0.35549 & 0.34473 & 0.81021 & $1.00(1)$ & $3.0(2)$ \\
\hline $\mathrm{C} 2$ & 0.45434 & 0.46446 & 0.81535 & $1.00(1)$ & $3.0(2)$ \\
\hline $\mathrm{C} 3$ & 0.54406 & 0.54911 & 0.77980 & $1.00(1)$ & $3.0(2)$ \\
\hline $\mathrm{C} 4$ & 0.45827 & 0.49626 & 0.86085 & $1.00(1)$ & $3.0(2)$ \\
\hline C5 & 0.55211 & 0.61990 & 0.88247 & $1.00(1)$ & $3.0(2)$ \\
\hline N6 & 0.36157 & 0.39433 & 0.88126 & $1.00(1)$ & $3.0(2)$ \\
\hline C7 & 0.33312 & 0.38937 & 0.92919 & $1.00(1)$ & $3.0(2)$ \\
\hline H71 & 0.40468 & 0.40097 & 0.94601 & $1.00(1)$ & $3.0(2)$ \\
\hline H72 & 0.31492 & 0.45507 & 0.93635 & $1.00(1)$ & $3.0(2)$ \\
\hline H73 & 0.26135 & 0.30887 & 0.93622 & $1.00(1)$ & $3.0(2)$ \\
\hline $\mathrm{C} 8$ & 0.30069 & 0.30373 & 0.84985 & $1.00(1)$ & $3.0(2)$ \\
\hline C9 & 0.18709 & 0.17522 & 0.85914 & $1.00(1)$ & $3.0(2)$ \\
\hline $\mathrm{C} 10$ & 0.31864 & 0.27574 & 0.76749 & $1.00(1)$ & $3.0(2)$ \\
\hline H31 & 0.58706 & 0.50928 & 0.76696 & $1.00(1)$ & $3.0(2)$ \\
\hline C11 & 0.17437 & 0.22503 & 0.76330 & $1.00(1)$ & $3.0(2)$ \\
\hline $\mathrm{C} 12$ & 0.12142 & 0.21818 & 0.71744 & $1.00(1)$ & $3.0(2)$ \\
\hline C13 & 0.14140 & 0.32353 & 0.74865 & $1.00(1)$ & $3.0(2)$ \\
\hline C14 & 0.01145 & 0.31775 & 0.74744 & $1.00(1)$ & $3.0(2)$ \\
\hline N15 & 0.00976 & 0.37627 & 0.70461 & $1.00(1)$ & $3.0(2)$ \\
\hline $\mathrm{C} 16$ & 0.00078 & 0.48146 & 0.69713 & $1.00(1)$ & $3.0(2)$ \\
\hline C17 & -0.00974 & 0.56345 & 0.73250 & $1.00(1)$ & $3.0(2)$ \\
\hline N18 & 0.00233 & 0.50585 & 0.65307 & $1.00(1)$ & $3.0(2)$ \\
\hline C19 & -0.00745 & 0.62038 & 0.64492 & $1.00(1)$ & $3.0(2)$ \\
\hline $\mathrm{C} 20$ & 0.01260 & 0.41437 & 0.63085 & $1.00(1)$ & $3.0(2)$ \\
\hline $\mathrm{C} 21$ & 0.01795 & 0.40234 & 0.58114 & $1.00(1)$ & $3.0(2)$ \\
\hline
\end{tabular}




\begin{tabular}{|c|c|c|c|c|c|}
\hline $\mathrm{C} 22$ & 0.01731 & 0.33240 & 0.66328 & $1.00(1)$ & $3.0(2)$ \\
\hline $\mathrm{C} 23$ & 0.02873 & 0.23076 & 0.63860 & $1.00(1)$ & $3.0(2)$ \\
\hline H32 & 0.49730 & 0.56338 & 0.75729 & $1.00(1)$ & $3.0(2)$ \\
\hline H33 & 0.60474 & 0.62836 & 0.79261 & $1.00(1)$ & $3.0(2)$ \\
\hline H51 & 0.63600 & 0.63374 & 0.87867 & $1.00(1)$ & $3.0(2)$ \\
\hline H52 & 0.53360 & 0.61655 & 0.91344 & $1.00(1)$ & $3.0(2)$ \\
\hline H53 & 0.54624 & 0.68784 & 0.86901 & $1.00(1)$ & $3.0(2)$ \\
\hline H91 & 0.20946 & 0.13139 & 0.88037 & $1.00(1)$ & $3.0(2)$ \\
\hline H92 & 0.16006 & 0.12726 & 0.83225 & $1.00(1)$ & $3.0(2)$ \\
\hline H93 & 0.11970 & 0.18550 & 0.87070 & $1.00(1)$ & $3.0(2)$ \\
\hline H101 & 0.36494 & 0.33268 & 0.74388 & $1.00(1)$ & $3.0(2)$ \\
\hline H102 & 0.33729 & 0.20830 & 0.76820 & $1.00(1)$ & $3.0(2)$ \\
\hline H111 & 0.13024 & 0.16224 & 0.78559 & $1.00(1)$ & $3.0(2)$ \\
\hline H121 & 0.03315 & 0.15668 & 0.71605 & $1.00(1)$ & $3.0(2)$ \\
\hline H122 & 0.19832 & 0.24190 & 0.70176 & $1.00(1)$ & $3.0(2)$ \\
\hline H131 & 0.20645 & 0.40776 & 0.75577 & $1.00(1)$ & $3.0(2)$ \\
\hline H141 & -0.05736 & 0.23235 & 0.74835 & $1.00(1)$ & $3.0(2)$ \\
\hline H142 & 0.00303 & 0.36486 & 0.77125 & $1.00(1)$ & $3.0(2)$ \\
\hline H171 & -0.00914 & 0.52999 & 0.76114 & $1.00(1)$ & $3.0(2)$ \\
\hline H172 & -0.08711 & 0.56448 & 0.72866 & $1.00(1)$ & $3.0(2)$ \\
\hline H173 & 0.06034 & 0.64788 & 0.73014 & $1.00(1)$ & $3.0(2)$ \\
\hline H191 & 0.06590 & 0.68161 & 0.62916 & $1.00(1)$ & $3.0(2)$ \\
\hline H192 & -0.08155 & 0.59822 & 0.62768 & $1.00(1)$ & $3.0(2)$ \\
\hline H193 & -0.01303 & 0.65545 & 0.67264 & $1.00(1)$ & $3.0(2)$ \\
\hline H 211 & 0.01298 & 0.46997 & 0.56701 & $1.00(1)$ & $3.0(2)$ \\
\hline $\mathrm{H} 212$ & -0.05158 & 0.32301 & 0.57170 & $1.00(1)$ & $3.0(2)$ \\
\hline H 213 & 0.09586 & 0.40641 & 0.57318 & $1.00(1)$ & $3.0(2)$ \\
\hline $\mathrm{H} 231$ & 0.03007 & 0.24524 & 0.60737 & $1.00(1)$ & $3.0(2)$ \\
\hline $\mathrm{H} 232$ & -0.04205 & 0.14959 & 0.64564 & $1.00(1)$ & $3.0(2)$ \\
\hline $\mathrm{H} 233$ & 0.10539 & 0.23299 & 0.64712 & $1.00(1)$ & $3.0(2)$ \\
\hline \multicolumn{6}{|c|}{$\begin{array}{l}\text { a symmetry operations used to generate remaining framework atoms and fluoride ions }(\mathrm{T} n: \mathrm{T}=\mathrm{Si} \text { or } \mathrm{Ge}, n=1 \\
5 ; \mathrm{O} n, n=1-12 ; \mathrm{Fn}, n=1,2) \text { : }\end{array}$} \\
\hline \multicolumn{6}{|c|}{$\mathrm{T} n 2:-x \mathrm{~T} n,-y \mathrm{~T} n, z \mathrm{~T} n+1 / 2 ; \mathrm{T} n 3: y \mathrm{~T} n, x \mathrm{~T} n, 2 / 3-z \mathrm{~T} n ; \mathrm{T} n 4:-y \mathrm{~T} n,-x \mathrm{~T} n, 1 / 6-z \mathrm{~T} n$} \\
\hline \multicolumn{6}{|c|}{$\mathrm{O} n 5:-x \mathrm{O} n,-y \mathrm{O} n, z \mathrm{O} n+1 / 2 ; \mathrm{O} n 6: y \mathrm{O} n, x \mathrm{O} n, 2 / 3-z \mathrm{O} n ; \mathrm{O} n 7:-y \mathrm{O} n,-x \mathrm{O} n, 1 / 6-z \mathrm{O} n$} \\
\hline \multicolumn{6}{|c|}{$\mathrm{F} 2: y \mathrm{~F} 1, x \mathrm{~F} 1, z \mathrm{~F} 1+1 / 3$} \\
\hline \multicolumn{6}{|c|}{ estimated standard deviations of refined parameters are given in parentheses in units of the last digit shown } \\
\hline
\end{tabular}




\section{Reference}

1. J. E. Schmidt, M. W. Deem, M. E. Davis, Synthesis of a Specified, Silica Molecular Sieve by Using Computationally Predicted Organic Structure-Directing Agents. Angew. Chem. Int. Ed. 53, 83728374 (2014).

2. A. Rojas, O. Arteaga, B. Kahr, M. A. Camblor, Synthesis, Structure, and Optical Activity of HPM1, a Pure Silica Chiral Zeolite. J. Am. Chem. Soc. 135, 11975-11984 (2013).

3. S. K. Brand et al., Enantiomerically enriched, polycrystalline molecular sieves. Proc. Acad. Nat. Sci. 114, 5101 (2017). 